Let's Go Steady: A Digital Communication Tool For Loving Couples by

Michael Layden Stevenson

BA, University of New Brunswick, 2009

Certificate in Film Production, University of New Brunswick, 2009

Diploma in Digital Design, Vancouver Film School, 2013

\author{
A Major Research Project and Paper \\ Presented to Ryerson University \\ in partial fulfillment of the requirements for the Degree of \\ Master of Digital Media \\ in the program of Digital Media \\ Toronto, Ontario, Canada, 2018 \\ (C) Michael Layden Stevenson, 2018
}




\section{Author's Declaration}

I hereby declare that I am the sole author of this MRP. This is a true copy of the MRP, including any required final revisions.

I authorize Ryerson University to lend this MRP to other institutions or individuals for the purpose of scholarly research.

I further authorize Ryerson University to reproduce this MRP by photocopying or by other means, in total or in part, at the request of other institutions or individuals for the purpose of scholarly research. I understand that my MRP may be made electronically available to the public.

Michael Layden Stevenson 
Let's Go Steady: A Digital Communication Tool For Loving Couples

Master of Digital Media, 2018

Michael Layden Stevenson

Master of Digital Media, Ryerson University

\begin{abstract}
As progressive societies increasingly rely on digital technologies, our methods of communicating with each other continue to evolve. While this opens up many possibilities for the formation of new relationships, it also has repercussions with regard to our ability to maintain the relationships that matter most. This paper and project aims to identify challenges experienced by couples in loving relationships typically caused by or worsened by over-reliance on smartphones and social media. I have proposed a design solution in the form of a mobile application made specifically for committed partners, tentatively called Steady. The goal of the app is to encourage compassion, understanding and healthy communication; helping couples be more engaged in face-to-face interaction and avoid being distracted by their devices while spending time together.
\end{abstract}




\section{Acknowledgements}

This project is dedicated to my parents, Donald Stevenson and Carolyn Layden-Stevenson, who provided me with a shining example of how to maintain a successful marriage throughout my life. They were absolutely dedicated to one another, and their love was unwavering even during times of great duress, or when their professional lives kept them physically apart. My mother is no longer with us, but I know she is proud of all my accomplishments, as is my father. I could not have gotten to this point without their continued support.

I would also like to thank several members of Ryerson University's faculty and staff for encouraging and supporting me throughout this project, which was not easy for me to complete based on the nature of the research and how it relates to experiences I have had in the past. In no particular order, I would like to extend a big thank you to Michael Carter, Sonya Taccone, Ahmed Sagarwala, Dr. Alex Ferworn, Mary Neelands, Dr. Richard Lachman, Dr. Paul Moore, Alevtina Naumova, Rob Blain, and Dr. Maria Chapparo. It has been my pleasure to learn from you all. 
Table of Contents

$\begin{array}{lll}\text { Author's Declaration } & \ldots . . . & \text { ii }\end{array}$

$\begin{array}{lll}\text { Abstract } & \ldots \ldots & \text { iii }\end{array}$

$\begin{array}{llll}\text { Acknowledgements } & \ldots . . . & \text { iv }\end{array}$

Table of Contents $\quad \ldots \ldots . \quad \mathbf{v}$

Body

$\begin{array}{lll}\text { Ch. } 1 \text { - Introduction } & \ldots . . & 1\end{array}$

$\begin{array}{lll}\text { Ch. } 2 \text { - Unavailable } & \ldots . . & 2\end{array}$

Ch. 3 - Somewhere Else $\quad \ldots \ldots . .4$

Ch. 4 - The Modern Digital Dating Landscape $\quad$..... 6

Ch. 5 - Similarities to "That Other" Dating App $\quad$..... 9

$\begin{array}{lll}\text { Ch. } 6 \text { - Sources of Income } & \ldots . . & 10\end{array}$

$\begin{array}{llr}\text { Ch. } 6 \text { - Conclusion } & \ldots \ldots & 12\end{array}$

Appendix

$\begin{array}{lll}\text { SWOT Analysis } & \ldots \ldots & 13\end{array}$

$\begin{array}{lll}\text { Site Map } & \ldots \ldots & 19\end{array}$

$\begin{array}{lll}\text { Wireframes } & \ldots \ldots & 19\end{array}$

$\begin{array}{lll}\text { Moodboards } & \ldots \ldots & 22\end{array}$

$\begin{array}{llr}\text { Style Guide } & \ldots \ldots & 25\end{array}$

Logo and Wordmark $\quad \ldots \ldots$.

$\begin{array}{lll}\text { Comps } & \ldots \ldots & 26\end{array}$

$\begin{array}{lll}\text { References } & \ldots \ldots & 29\end{array}$ 


\section{Ch. 1 - Introduction}

Over the past decade, our methods of communicating with each other have evolved tremendously due to advancements in digital technology (Hertlein \& Piercy, 2008). Innovations in Short Message Services (SMS) and Social Networking Sites (SNS) such as Facebook have enabled us to remain in constant contact with our ever-expanding professional and social networks. This has opened doors for professional and personal relationships to be built and maintained across vast distances, among people who may not have had a chance to interact otherwise. However, these advancements also create unforeseen consequences with respect to our ability to engage in meaningful face-to-face interaction. This is particularly worrisome with regard to younger generations who have grown up in the digital age and do not possess an adequate frame of reference from which to draw comparison. Many academic studies have examined the ways that over-dependence on mobile devices can negatively affect our ability to maintain focus for long periods of time (Coyne, Stockdale, Busby, Iverson, \& Grant, 2011; Henline, 2006; McCormack, 2015). However, only recently have these concerns permeated into mainstream culture, with popular online blogs like BuzzFeed and Huffington Post now frequently posting stories about the dangers of smartphone addiction (Wong, 2017). Even less attention is being given to the ways in which the dating landscape has changed through the popularization of applications like Tinder, Bumble and Grindr. This is concerning because there is little doubt among users of these apps that the technology has a tendency to change the way we think about potential partners and commitment. We are exposed to a variety of platforms to aid us in our search for the 'perfect match'. However, these services only exist to make a first introduction between you and another person. After that, you and your partner are on your own. Apps do not check in with you a month or year down the road to see how your relationship is going and make suggestions regarding what you could do better. 
Our reliance on technology to keep us organized, informed and entertained has increased significantly. Many young adults feel overworked and generally too busy to enter into a dedicated relationship with one partner (Notable Life, 2015). Instead, they are often content to simply 'play the field' and dismiss any feelings of yearning for something more (Lyadova \& Hughes, 2017). We live in an age of efficiency, and use technology to get the most satisfaction out of a product or service while exerting the least amount of effort possible (Grinols \& Rajesh, 2014). When this mentality translates to dating, there are plenty of opportunities for failed communication and unhappiness. Lasting relationships take a great deal of work on both sides, with compromise being key. Selfishly expecting to get more out of a relationship than you yourself put in is a recipe for disappointment. However, because dating apps can create the illusion that there are an unlimited number of fish in the sea and that 'the grass is always greener on the other side', people with all levels of dating experience have a tendency to resist these notions of chivalry, and often give up on budding relationships at the first sign of trouble (Nahai, 2017). With the rise in popularity of digital tools for a variety of self-help initiatives (ex. monitoring health and keeping a daily exercise routine), I believe now is the perfect time to release a new type of dating app to the general public - one that has been designed from the ground up for those who are already in a committed relationship. Tentatively titled 'Steady', my proposed design takes into account the negative tendencies associated with mobile devices, and aims to streamline the communication process between loving partners through user experience (UX) and human-centered design philosophies and techniques (Thomsen, 2013). Emphasizing compassion and compromise, Steady has the potential to help couples identify problematic tendencies in their smartphone use, and work toward eliminating these tendencies and enjoy stronger, more exciting relationships.

\section{Ch. 2 - Unavailable}

As digital technology becomes increasingly affordable and user-friendly, more people are able to use it to complete a greater variety of tasks. Facebook, formerly a haven for young adults, is now 
populated with as many grandparents and pre-teens (Dawley, 2016). Many simple daily tasks that used to be performed manually can now be accomplished online with much less effort, such as transferring money between bank accounts, holiday shopping or requesting time off work. Even the most complex creative projects with large teams can be completed collaboratively over the Internet using project management tools and shared software (Wells, 2014). However, these benefits come with a cost. An increase in the size of our communication networks means an increase in places to go and people to see. While we can use technology to accomplish more in a given amount of time through multi-tasking, we are now connected to social and professional networks that ask we remain digitally available at all times. A day off is not always truly a day off, as we often have emails to catch up on or notes to prepare. Many young professionals carry their smartphones with them everywhere they go constantly 'on-call' in preparation for the inevitable event a where colleague or client has a concern. This leaves very little room for building upon a new romantic relationship, let alone finding 'the one' while maintaining a fast-paced professional lifestyle. With so many events to keep track of, we often need digital calendars to help us remember. They fill up quickly, even for a single adult with no children, and maintaining one can be a challenge. Compare this with the much more difficult task of maintaining a relationship on two very different schedules. Misaligned free time can lead to frustration. Established relationships can compromise and persevere through these times. For newer romances, however, frustration might lead to poor communication or distaste, which may intensify the problem. Intimate relationships, both young and old, could benefit from a way to organize time so that time spent together is better recognized. Married couples likely know each other's schedules already, but it can still be easy to forget who is supposed to pick up the kids from school or when to call the babysitter and take a night off. When asked why they are single, many young adults cite lack of free time as a reason, especially for those who travel extensively for work (Rendón, 2015). This is the primary source of inspiration for the first major design aspect of Steady - the "Somewhat Shared Calendar". Although planning dates can be a struggle with multiple time commitments, privacy 
concerns prevent many couples from using shared calendars. However, with API aggregation technology (Boyd, 2013), this feature simply needs to be implemented with privacy in mind, first and foremost. Steady will implement smarter scheduling for couples, as users can link their respective calendar apps and have the aggregated data overlap on the back-end. Sensitive information is kept confidential, while each user views a simplified display with suggestions for optimal times to get together based on a compromise between schedules. By emphasizing mutual free time, finding the right moments to relax and enjoy each other's company will be simpler. Users can browse through appropriate time blocks, select a desired time and begin browsing through potential activity ideas. These events are populated from Facebook's API in order to make worthwhile suggestions based on users' tastes. Mutual interest in an event prompts a notification in the same way a dating app would display a match, and provides all relevant information with links to purchase tickets and get driving directions. The process of finding a suitable time and choosing an activity is not only made more efficient, but also creates a fun cooperative experience to help couples learn more about each other and increase anticipation. Reminders can be set in case of a lapse of memory, but this in itself presents a new problem.

\section{Ch. 3 - Somewhere Else}

In progressive societies, digital distractions are a constant reality. Streets and restaurants (and many homes) are packed with flashing images. Our inboxes update relentlessly, phones buzzing in our pockets with a blend of professional, social and promotional reminders. Our devices serve as distractions rather than tools of efficiency, which can lead to difficulty focusing and maintaining good communication in real-world relationships (Roberts \& David, 2017). Digital notifications do not care if we are making a conscious effort to remain 'present in our current moment' or not. For many who are trying to establish themselves professionally in an increasingly competitive environment - we have to check them anyway, just in case it happens to be a crucial work email. We have all apologized as we are 
pulled away from a romantic endeavor because of a digital notification, and we have all been on the flip-side, assuring our date, and ourselves, that "It's ok, not to worry". A 2012 Essex University study noted "the presence of a diverting influence such as a mobile phone may inhibit relationship formation by reducing individuals' engagement and attention for their partners, and discouraging partners' perceptions that any self-disclosure had been met with care and empathy" (pg. 239, Przybylski \& Weinstein, 2012). Consistent attachment behaviors displayed through texting, liking and commenting have been shown to have a direct correlation to relationship satisfaction (Coyne et al, 2011; Pettigrew, 2009). Yet the very device we use to stay connected is also heavily linked to feelings of envy, detachment and isolation (Appel, Crusius, \& Gerlach, 2015). Smartphone addiction is becoming increasingly common, and afflicted persons can show patterns similar to those addicted to substances, ignoring responsibilities and loved ones in favor of a gateway to the Internet (Prizant-Passal et al, 2016). Once the planning phase is finished and a date is underway, phone use should (ideally) be kept to a minimum so that mutual engagement can occur freely. Sometimes it is necessary to check an email from work, but ignoring a partner for extended periods of time is bound to lead to dissatisfaction. For some, turning off your phone or leaving it tucked away can be a great way to counteract this. For others, this is simply not an option. Steady will use Near Field Communication technology (NFC) (Blue Bite, 2017) to implement a design solution that tracks device usage and sets friendly goals for minimizing it during designated times when partners are together. NFC technology will determine when partners are in the same room, and Steady will offer different options for setting boundaries on phone usage while spending quality time together. This feature, called Brick Mode, has three settings for removing the distracting elements of smartphones during quality time. The Light setting will prompt users after a handful of phone activations during an allotted time, using a 'colorful tone' to remind them that they ought to pay more attention to their dates. The Medium setting requires a correct response to a trivia question (entered by a user's partner) before problematic apps can be used, while Heavy prohibits both parties from accessing these apps until both simultaneously agree to 
lift the restriction. Rather, it exists as a way to encourage partners to keep their phones at bay, but can also result in light, healthy arguments (ex. using the Medium setting, a boyfriend might be unable to check his favorite sports team's statistics while on a date because he cannot remember his girlfriend's favorite animal). It is important to note that Brick Mode will in no way interfere with smartphones' built-in Emergency Call functionality, as doing so would present a serious concern regarding personal security.

Also important is the question of how much messaging is too much while apart. No partner wants to become the distracting notification for his or her beloved. Once Steady has accumulated a user base and gone through various forms of testing, the aforementioned Somewhat Shared Calendar feature could be used in conjunction with more prompts or suggestions to great effect. For example, Steady might remind Harry that his girlfriend Sally is studying for a big exam on Wednesday night, so it might not be the best time to message her repeatedly. Not only does Harry avoid becoming a distraction, but he also finds the perfect time to wish Sally good luck the following morning. With better guidance (and more metadata), we can communicate more effectively, reducing the risk of becoming distracted. The harsh reality is that these minor distractions, if unchecked, can build up over time and eventually become significant concerns for both parties. So much so that people might begin to explore other options, of which there are (seemingly) infinite.

\section{Ch. 4 - The Modern Digital Dating Landscape}

Years ago, a typical first date might be preceded by a number of conversations or advances. In the post-Tinder world, we have the ability to go on multiple dates with complete strangers within the span of a day if we so choose. We have criteria for choosing mobile dating applications based on our communication preferences. If you think women should be the first to type a message after matching, try Bumble. Or if you want the run-of-the-mill (mildly shallow) online dating experience, Tinder is a safe bet, with 10 million daily active users spending an average of 35 minutes 
swiping per day (Smith, 2018). However, time spent on dating apps that focus on instant gratification might not be good for users' self-esteem, or their ability to form meaningful romantic connections in the future. Swiping left/right to either Like or Dislike a person's profile has widely been compared to window-shopping, or "dreaming of what might have been or what could still be" (Matyszczyk, 2015). Matches are at the same time idealized and devalued (Hilton-Dunne, 2016). Users have a tendency to match with many people at a time, often without ever messaging any (Weiss, 2016). This could be due to a lack of genuine interest, a subconscious anxiety, or something else entirely. Reports have shown that only approximately 54\% of Tinder users are actually single (Smith, 2018; Matyszczyk, 2015). This sparks a debate around whether matching on Tinder out of boredom while already in a relationship constitutes 'cheating' or not. Users ultimately decide how they will or will not use dating apps. These apps are not inherently negative, but they do have the potential to create problematic behaviours (Schade et al, 2013). Frequent disappointment found in not matching with other users can lead to a negative self-image, which in turn can make it more difficult to feel confident sending a first message. On the other hand, having an extensive list of matches creates an illusion of potential mates waiting to be swept off their feet. A user with this sort of 'romantic waitlist' might become unable to accept any small issues in a current relationship because of expectations that the perfect situation can be found right around the corner, with a more compatible partner. Many people who make a genuine attempt at using casual dating apps to find a sincere partner quickly become frustrated and abandon their quests (Nahai, 2017). This is often caused by the number of matches who are either inappropriate in their messages, or fail to respond entirely. Even if a user has success in finding matches, the process of filtering through these and separating the worthwhile from the worthless can be taxing, especially for those who keep traditional views on relationships.

In 2016, a Toronto woman made popular blog headlines when she outsourced her online dating profile to an employee based on an hourly wage (Strapagiel, 2016; Vendeville, 2016). Katerina Lyadova, a young entrepreneur who manages her own creative design company, sought to eliminate 
the hassle and frustration of dealing with an over-population of matches that failed to meet expectations. In a thought-provoking experiment, Lyadova posted an ad on Craigslist in search of a Dating Profile Manager. She eventually found suitable candidates, who she paid for roughly 7 working hours per week to swipe and chat with new matches on her dating profiles, eventually setting up first dates. If Lyadova considered a date to be a success, her employees would receive a bonus. Upon exchanging phone numbers with a date, a transition would occur where the man in question would now be in contact with Lyadova herself, rather than her employee (as it had previously been through Tinder messages). The experiment lasted three months, and ended up with Lyadova publishing a book with the help of Melissa Hughes (a copywriter at her company at the time), called Dating Vandalized (Lyadova \& Hughes, 2017). The book, a work of fiction based on true events, is an engaging introspective into the personal life of a young, successful woman looking for love in an increasingly complicated dating landscape. Unfortunately, the experiment did not make the dating scene in Toronto any easier to navigate for Lyadova, as she notes that she found her most notable romantic connection without the help of any dating app or digital tool (Lyadova \& Hughes, 2017).

"As a designer, I marvel at the algorithms, the UX behind these apps. It can be viewed as astounding progress, humanity using tech to connect. And yet I feel a disconnect. It started before the world went digital, blossoming in the crush of cities, in the hustle of 95 , in the isolation of the post-industrial era. We must cope with density, volume, abundance - and also with the vacuum it creates. Are the tools we use - Tinder, OkCupid, Bumble, happn - problematic, or should we look, instead, at the conditions that necessitate them? We build what we need. We use what we must. Resistance is optional" (pg. 134, Lyadova \& Hughes, 2017).

Keeping in mind the question of morality surrounding the various ways that dating applications are being used, it becomes a question of which came first - the chicken or the egg? Does spending too much time on Tinder have the potential to morally degrade users, dooming them to eventually become more shallow, judgemental, self-conscious and deflective people? Or is the popularization of these apps merely the inevitable bi-product of symptoms that have already existed in our subconscious for who knows how long? This is too broad, too deep a question for the purposes of this paper. However, it is 
difficult to deny that despite best efforts, many Tinder users do not get the results they are looking for from casual dating apps. As mentioned previously, problematic cellphone usage is particularly concerning with regard to younger generations. Parents are often the first to notice signs of detachment and isolation in their children as a result of too much time spent on digital devices (TackieOfosu, 2015). And yet Tinder's design uses gamification to increase the addictive qualities of the swiping process (Nahai, 2017). Matching with another user offers a brief period of excitement. Perhaps this is why many users spend so much time matching without following through and messaging their matches. It could also be why people continue to use dating apps even when they are already in a relationship.

Having a place for couples to go to revisit past memories and blueprint the future could help to combat this mentality. Steady will use NFC to create a collaborative digital scrapbook that populates automatically with photos taken while together. This would remove the need for any upkeep and keep phones out of sight more often, reinforcing quality time together. Every viewing becomes fresh and new, and serves as a worthwhile distraction in place of browsing through other fish in the sea.

\section{Ch. 5 - Similarities To "That Other" Dating App}

A portion of Steady's User Interface intentionally borrows from popular dating apps like Tinder for a variety of reasons. These conscious design decisions have been put in place to make the transition from a 'dating app' to a 'relationship app' seamless for newer couples that are familiar with current social media technology. It was also designed this way to call attention to the psychological shifts that can occur as a result of these apps becoming the norm (Finkel, Eastwick, Karney, Reis, \& Sprecher, 2012; Wong, 2016). Not looking to pass judgment, Steady seeks to provide users with healthy alternatives to problematic behaviours. In a way, this project is an attempt to remind the user that relationships take a great deal of effort and compromise to make work, and assist in making the digital side of communication as easy and fun as possible. Swiping left and right on potential date ideas 
for you and your significant other to enjoy together is a much healthier activity for those already in a relationship than browsing through 'other fish in the sea'. Moreover, the dating process is made more cooperative and interactive for both partners, with each person's interests holding as much weight as his or her partner's. Rather than getting bored of doing the same old things over and over, couples receive new suggestions on a consistent basis, so there is always something new and exciting to try. For a more complete description of all the features that Steady will implement in order to help couples achieve a balanced, healthy relationship, please refer to the UX documentation provided in the Appendix.

\section{Ch. 6 - Sources of Funding}

Steady certainly needs an influx of funding to pay for development and marketing costs in order to get off the ground. Once a Minimum Viable Product is established and people have a chance to see the product's value for themselves, however, I believe the user base will grow quickly and sources of funding will be limited only by the creativity of the project team. Online crowd-funding services like Kickstarter and Indiegogo would serve as a great way to introduce potential users to the platform and build interest. In order to mitigate any risks, Steady should only be promoted through these types of services after the code for the MVP has been finalized (and patented, where possible) and design elements such as the company name and logo have been copyrighted. In order to get to this point, then, I will initially apply for various grants and seek out private funding. Taking on a business partner could be markedly beneficial, particularly someone with masterful coding abilities who believes in the platform and would contribute his or her skills based on a partnership model that allocates shares in the company rather than a base salary. Once the aforementioned milestones have been met, Steady will be ready to be showcased to the public in full force. Throughout my process, I have received overwhelmingly positive feedback regarding the app's potential utility in real-world scenarios featuring real couples. Therefore, it is my belief that I will have no problem repaying any debts that the 
project may have accrued whilst getting ready to launch. Kickstarter offers donors different levels of rewards based on the size of their contributions. Steady can offer free trial subscriptions for small donations, and longer-term subscriptions in combination with merchandise and personalized rewards for more generous contributions.

Once Steady is available on the App Store and Google Play, there will be various sources of income that will ensure its growth. It makes the most fiscal sense to allow users to download the app free of charge for a trial period, with an inexpensive subscription model to be put in place after this trial ends. An alternative to this would be offering a Basic version of the app for free, with user subscriptions unlocking more advanced features. However, this would require enough user-testing to determine which features should be included in which version, and is therefore less likely to be proper in the beginning stages. Subscriptions prices would be minimal, from $\$ 0.99-\$ 2.99 /$ month. If this seems expensive, consider that many users of dating apps pay in excess of this rate to unlock the Premium features of those services, and that relationship counselling is much more expensive still. It is not difficult to imagine a couple experiencing relationship problems that would not be willing to try a beneficial app for a few dollars a month rather than going straight into counselling.

This subscription model serves as a good starting point, but as Steady's user base grows, the potential for advertising revenue becomes increasingly apparent. Events that show up in users' feeds can be sponsored by any number of clients looking to market their experiences to larger audiences. Sweepstakes could be implemented in different forms based on donations from sponsors. A free dinner for two could be randomly awarded to a couple that shows interest in the displayed Event for dining at a particular restaurant, for example. On a much larger scale, Steady could offer an allexpenses paid vacation to a randomly selected couple for sharing a promotional post while using hashtags and tagging friends. This would provide a huge incentive for users to promote Steady on their social media channels, in turn garnering more attention and bringing in more subscriptions. As Steady grows, so too will the opportunities for revenue and investment, allowing the company to hire more 
employees and continuously improve the product, with the needs of its users being considered first and foremost.

\section{Ch. 7 - Conclusion}

Evolutions in technology have changed the dating landscape in recent years, and have contributed to new problems in communication. Sherry Turkle (pg. 12, 2011) argues:

"As we distribute ourselves, we may abandon ourselves. Sometimes people experience no sense of having communicated after hours of connection. And they report feelings of closeness when they are paying little attention. In all of this, there is a nagging question: Does virtual intimacy degrade our experience of the other kind and, indeed, of all encounters, of any kind?"

I believe that digital communication technology does indeed degrade our face-to-face communication skills over time, as well as our abilities to show empathy and stay focused. However, it does not have to; the problems I have outlined in this proposal exist due to design flaws in our digital tools that have negative effects on the way we use them. A problem with dating applications is that it causes people to resist the fact that relationships take a lot of work and compromise, instead fooling themselves into believing that the grass is indeed greener on the other side (Sam Chan, 2017). I have proposed the development of a new application that I believe needs to be released - a dating app for those already in a relationship. Improving digital communication between romantic partners requires excellent UX design skills, encouraging more time for purely face-to-face interaction and less time on devices. The paradox of designing something that, if used effectively, reduces the necessity of its own use has been tricky to navigate. However, I believe I have achieved a compelling product design with the goal of helping couples be a little more compassionate, and a little more engaged with each other in the face of distraction. In the coming months, I aim to source enough funding to make this project a reality. I wholeheartedly believe in Steady's ability to provide assistance for couples who could benefit from it the only question is whether I can find myself in a position to take advantage of this opportunity before another (larger) company develops a worthy competitor. 


\section{Appendix}

\section{SWOT Analysis}

\section{Steady}

\section{SWOT Analysis}

\section{Google Calendar}

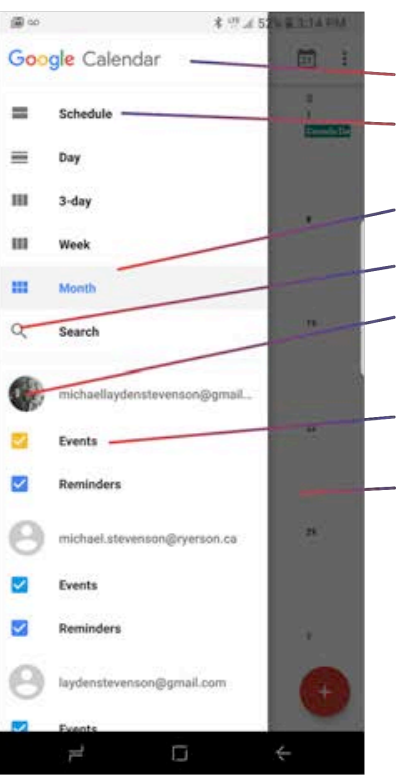

Primary Navigation/Contents

rimary navigation is accessed in the familiar manner of tapping (1)

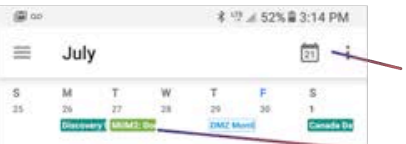

Month View

coogle Calendar's navigation is simple, and acts more like a filter w sual representatition to different periods of time that can be easily inderstood

Good use of bright color and subtle background change to highlight

Search located at the bottom of this section. Perhaps this would

xxt section allows user to filiter through different email accounts. whether the user sees all upcoming Potential Dates, only confirmed
Dates, or to also include all activities logged within Google Calendar

changing color to represent different types of activities. This (eative should be ponted to rep

Contents tab sildes out from left-hand side, and the background (screen that was being viewed previously) is greyed out. This helps
sers understand where they are in the application, and that they

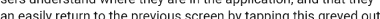
Jea, or the Back arrow icon at the bottom of the screen

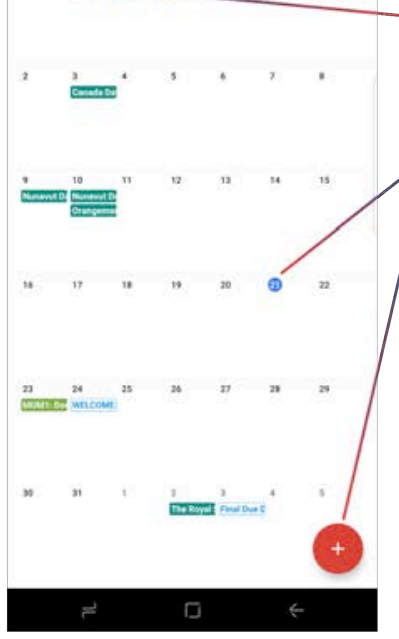

Function of these buttons is not clear. Icons for "Jump to Today" (a) destinations, such as Today, Last Date and Next Date, etc

Different colors used for different types of activities is nearly essential. In Steady's case these colors might be predetermined for within the application. Potentialy toggling fifter tos sow all activities recorded within Google Calendar would use the appropriate colors selected by the user within that seprate
application This could have negative effects for the readability of reen, or the overall aesthetic.

Today's date is highlighted with bit of color. However, this could

Large CTA button (colored differently than anything else on the expands upon tapping to reveal 3 different types of activities. Goals, Reminders and Events. Steady could use a similiar teature but perthaps only for Reminiders, as Potentitial and Confirmed Dates

Months View is well organized, considering the amount of Information hat cant be displayed on the scieen a tone time. unnecessary to include a full-screen Month View. It may make mere sense to have the screen split into 2 areas, top and bottom, one of which shows all the days in the month with the ability to highlight betrom the of the screen tos appropriate week and showing a simplified view with more room to Include time of day, etc.

\section{Steady}
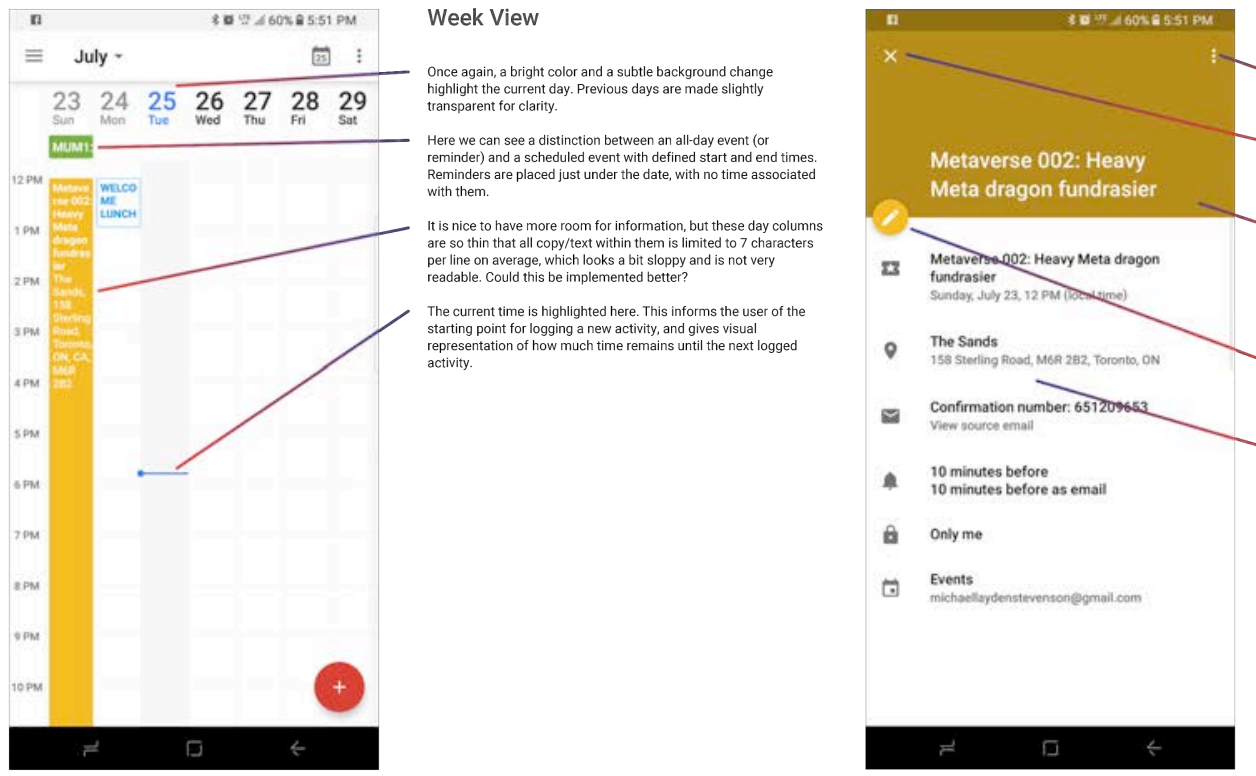

Single Activity

In this case, this dots icon only has an option to delete this entry. seems redundant and could potentially be removed.

Similarly, this exit button is made redundant by the Android OS's Back button at the bottom of the screen. However, as this may this exit button alone.

This top section could potentially be a missed opportunity. It uses a solid color instead of any image related to the event. Given th
tickets to this particular event were purchased through its on Facebook event page, and all relevant information was aggregated automatically, it seems silly not to include the provided event photo

Tap this button to edit the information associated with this entry (in especially if plans change suddenly.

Direct links to Google Maps and GMail a Only me

7 Events 


\section{SWOT Analysis (cont.)}

Steady



Split View - Month/Week

This screen was initially set to Week View. Tapping the arrow icon information further down the page. This is a useful feature the would translate very well to Steady, as it separates the screen into one part that helps you locate the time you are looking for, and the work well enough that this format could become the stand and the most visited section of the application.

Users are able to swipe left/right in this section to move tack/forward through months in the calendar. However, this is not explicitly communicated
make this more obvious.

Little colored dots can be seen below each date, showing the number and type of events/activities that a user has planned for additional feature added to show Past Dates, Future Potential Dates and Future Confirmed Dates. These could potentially be displayed circe shoun tere to diplat the curent
SWOT Analysis

Google Calendar

\section{Steady}

\section{Strengths}

- Consistency with all of Google's apps and platforms makes the Ul easy to learn
- Simple UI with minimal use of

- Limited use of color makes CTA's easily distinguishable

- Ability to manage multiple accounts simultaneously, switching between them on the fil

- Types of calendar entries are distinguished by color, and these colors are displayed in a very small and unobtrusive, yet readable way - Most buttons are large enough to tap easily without error

was essed through Primary Navigation

- Day and time indicators give schedule pages added readability

- Provide direct links to Gmail (confirmation/receipt emails) and Google Maps
- No clutter or excess present in single activities, only the necessary information is provided

\section{Opportunities}

-Use Google's Ul as a basis for experimentation - Staying true to Google UI makes the platform simple and easy to learn for users already familiar with Google's apps - Use subtle visual indicators (such as little colored dots or hearts) to distinguish between different types of activities - Dual-screen layout could show an entire month alongside a single week to convey as much information as possible with minimal screen clutter or potentially confusing elements
- Merge all types of data entry into one expandable button

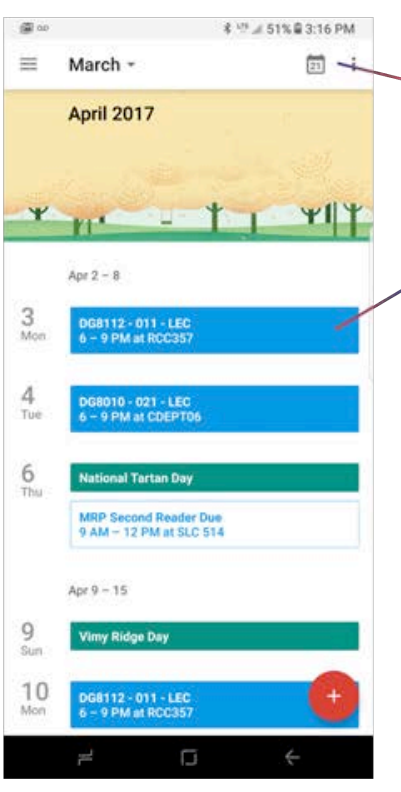

List View

This List view is similar to the Mermory Wall/Feed section that displayed, with all excess space or clutter removed. Users could swipe from month to month similar to what was shown in the Dess and Futren This negates the need for togging between Past The current month The user would be able to swipe left to go back in time, or right to review upcoming activities. The ability to quickly navigate to a month farther back in time could be implemented into

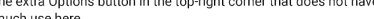

Tapping each entry would lead the user to a new screen with more in-depth information regarding this activity. Website and ticket links, photos, online reviews, etc. could all be used to give provice
more insight into a potential date idea.

\section{SWOT Analysis}

Google Calendar

\section{Weaknesses}

-A few redundant/unnecessary buttons and links could be merged together
-Small visual indicators could be made a bit more noticeable

Long, thin columns make text paragraphs unreadable and unattra

Missed opportunity not automatically a 


\section{SWOT Analysis (cont.)}

Steady
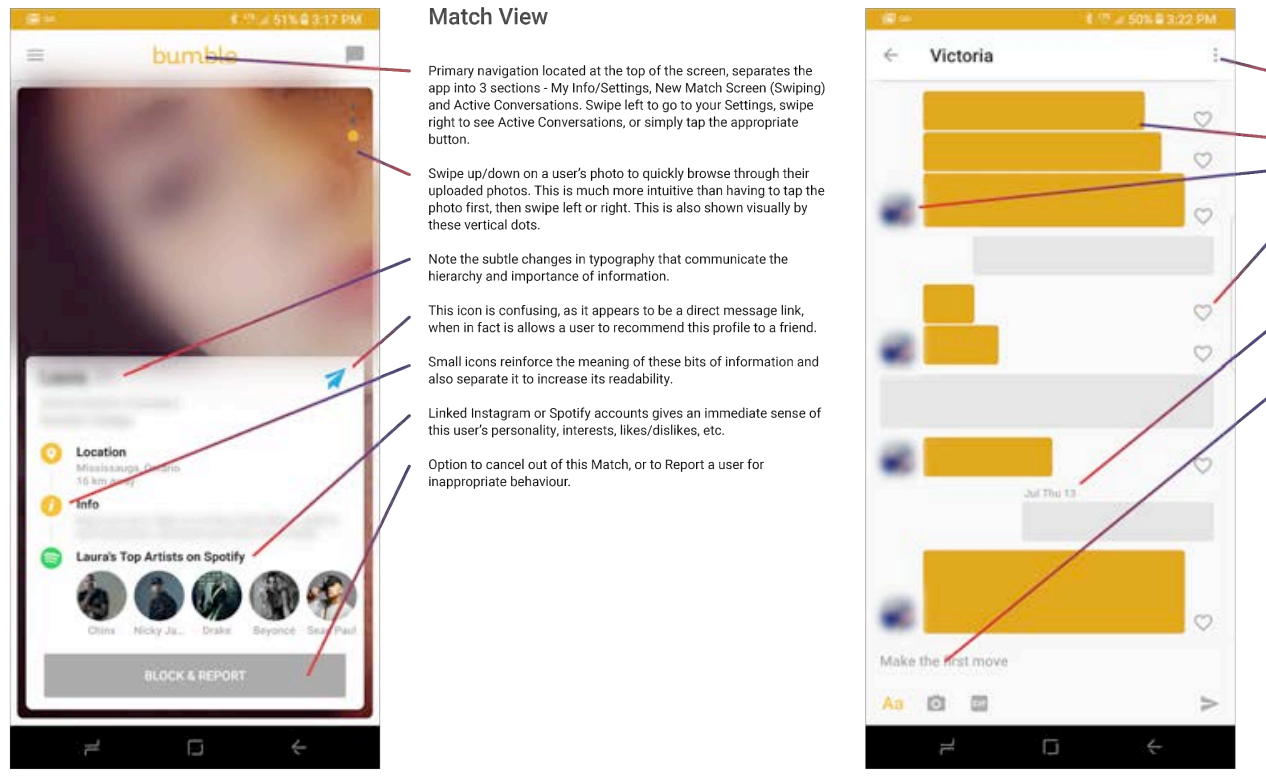

Conversation

Extra functions for View Profile, Block \& Report and Unmatch are located here. User can also visit a matchis profile by tapping

Difference in color helps easily distinguish who typed what Thumbnail photo constantly reminds user of who he/she is speaking with, and also helps to break up the information on the
screen so that who is typing what is even more clearly identifiable. Ability to like a match's message, similar to giving a Thumbs Up Facebouk Messengee. Could be used for Sheady users to "Favorite" a partner's
section.

Date is displayed here to differentiate between days. № added functionality such as swiping on a message to see the exact time it was sent.

Standard text entry field contains flavor text (nice touch there). Quick link
single tap.

\section{Steady}
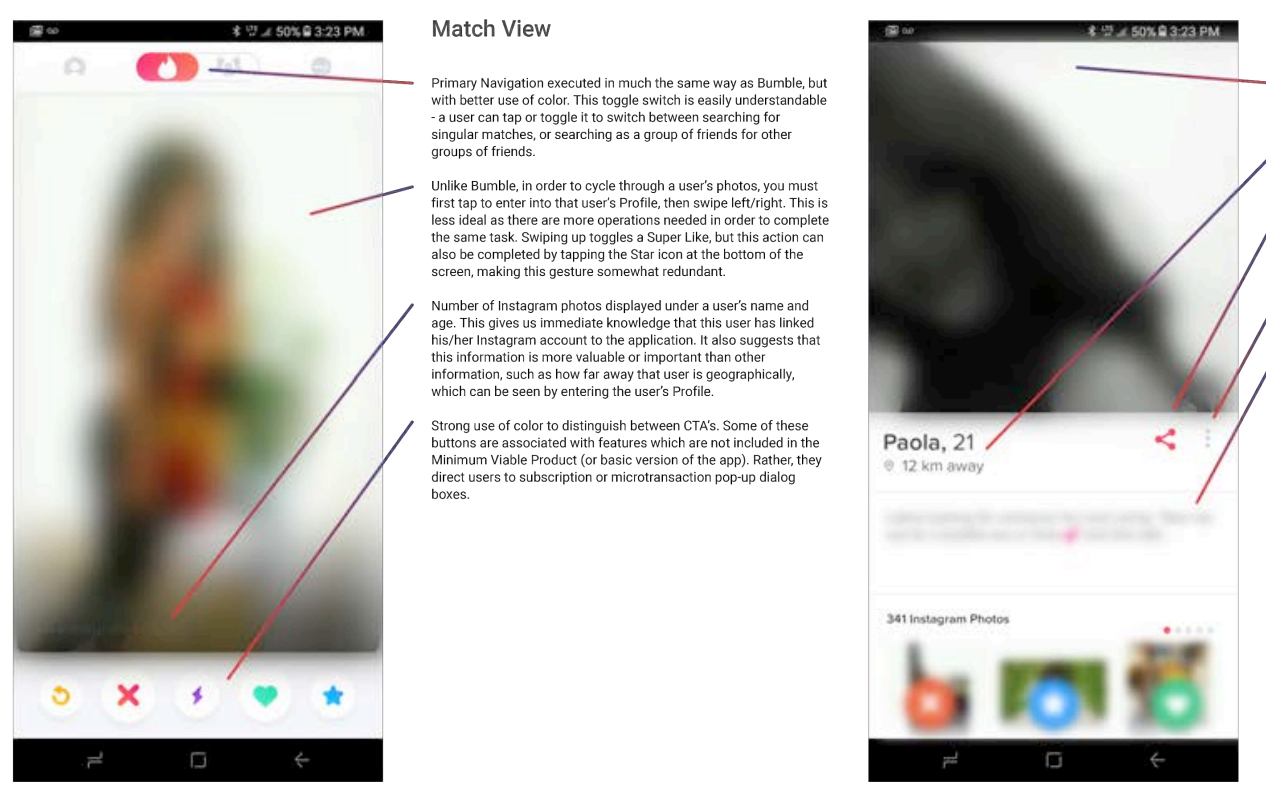

Profile View

Horizontal dots provide feedback on cycling through a usel's
photos - how many there are in total, and which is currently Number of Instagram photos has been replaced here by
geographical proximity, while Instagram has been given its own section at the bottiom of the screen.

This button allows a user to share another user's profile with friend, through a variety of apps and functions. For Steady's purposes, this could be used to recommend a date idea to a user's partner, or to another couple entirely.

Additional actions such as Unmatch, Block/Report user are located

Small space to insert some flavored text. For Steady's purposes, says, what their partner's description says, or both? The sapptions question arises with regard to a user's profile photo. 


\section{SWOT Analysis (cont.)}

Steady

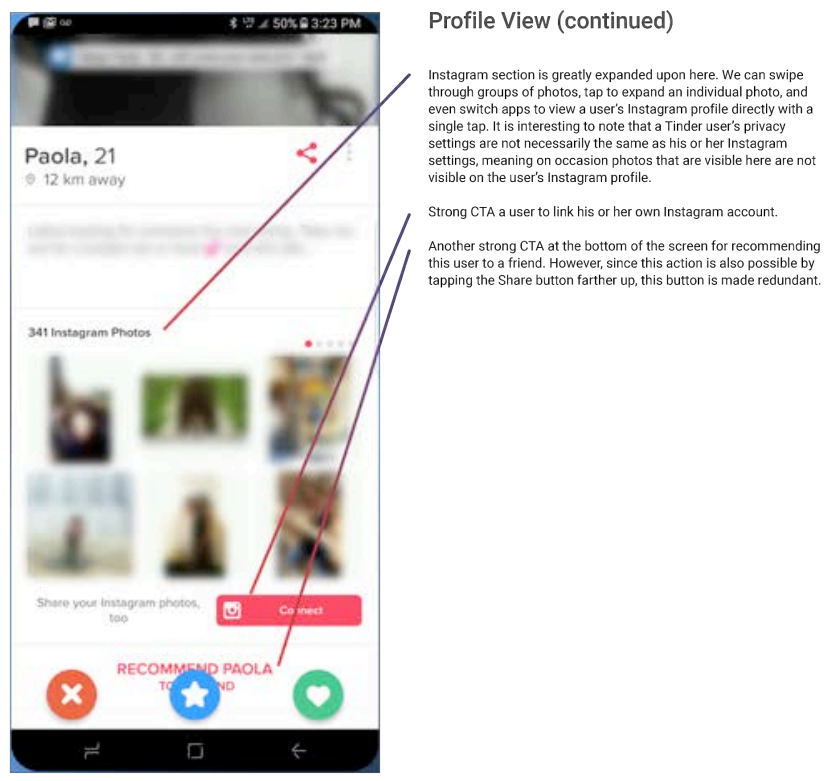

\section{Strengths}

- Simple Primary Navigation breaks the apps into 3 basic sections, is located at the top of the screen and is easy to use

- Use of colored/gray dots 1 go gesture control, ability to swipe in all 4 directions is useful

- All additional, rarely used links and actions are grouped together under a single icon

- Large sections complete with images for linked social media accounts such as Instag

- Toggle between two modes of search by tapping, this button's use is communicated visually (skeuomorphism)

- Easily attach images, Girs or inks to your messages (quickiliks)

\section{Opportunities}

- Design of swiping left/right could be translated to finding the optimal date idea once a suitable day and time has been selected,

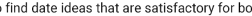

ork well for switching between different sections of Steacy

- Easy to link social accounts - directly travel to your partner's profiles on different social media (also enables features such as listening

to music or watching a video simultaneously, even when apart)

\section{Weaknesses}

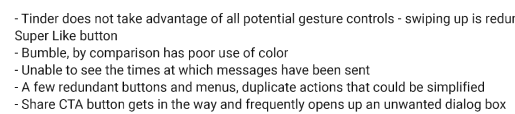

A few redundant buttons and menus, duplicate actions that could be simplified

\section{Threats}

Over-reliance on the swiping left/right gestures in the UI could alert users to the similarities to dating apps and draw criticism 


\section{SWOT Analysis (cont.)}

Steady

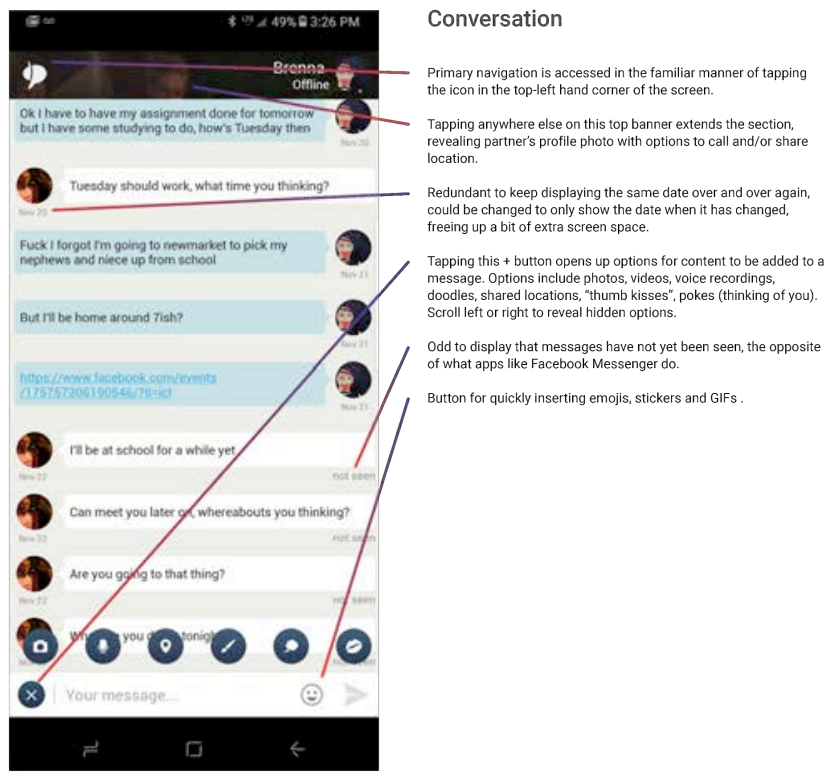

SWOT Analysis

Couple

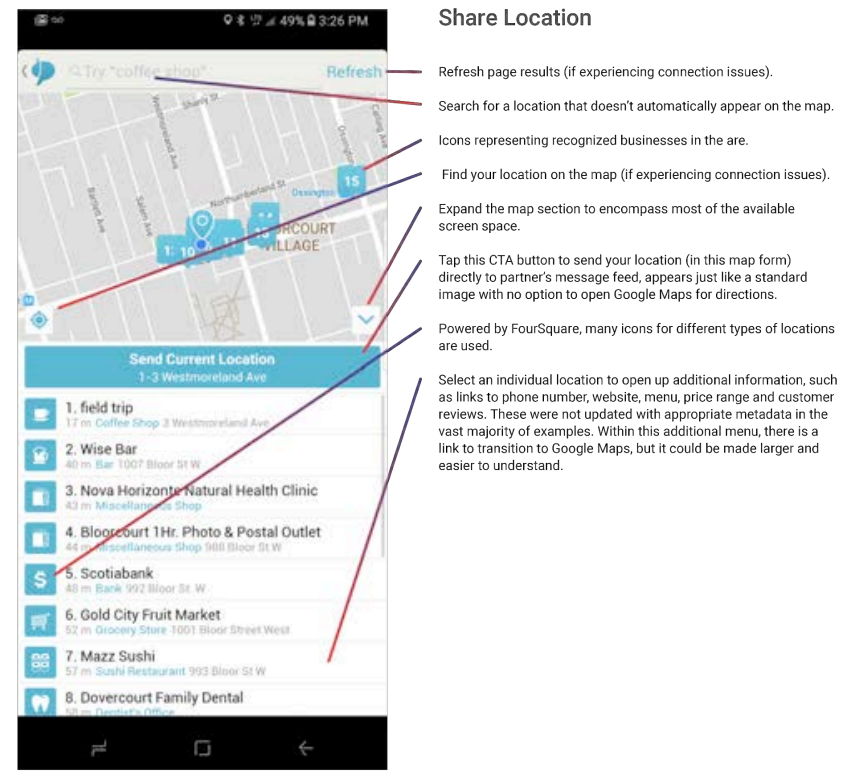

$9 / 12$

\section{Steady}

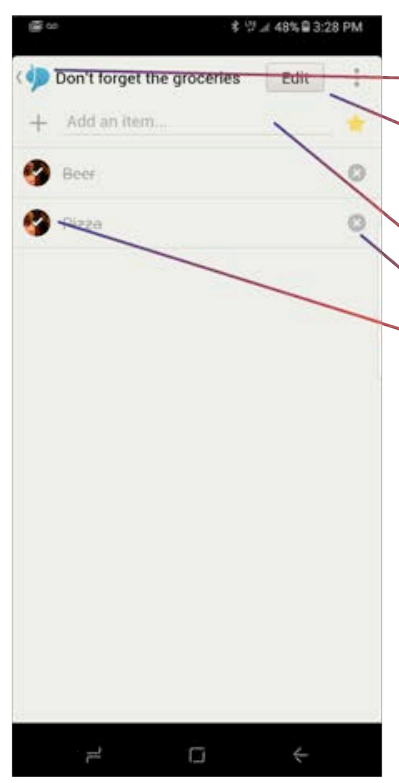

SWOT Analysis

Couple

Shared List

Back button is slightly redundant on Android OS, but this is not necessarily the case with other devices.

List title, with an Edit button and a drop-down list with the sole dded to the Edit menu. The Edit menu is saved from redundancy only because of the option to alter list items' spelling, correcting

Add a new item to the list, with an option to assign additional
priority in the form of a star marker.

Once an item has been completed, users can then remove it from the list by tapping this $\mathrm{x}$ button (duplicate action also available in
Edit menu).

Tapping a list item crosses it off the list, and also displays which

Important Dates

Add a new important event to remember. This could be a birthday, anniversary or a reminder to complete a certain errand. Events are gesture functionality implemented. Perhaps swiping left/right to scrict

Important information is displayed right away. Unsure why the event tite is grey and not bold black. Good to have the title and iconography to spice up the display of the date a little bit

Tap an event to edit its details. Change the date and time, add reminders and determine whether It is to be repeated on a yearly

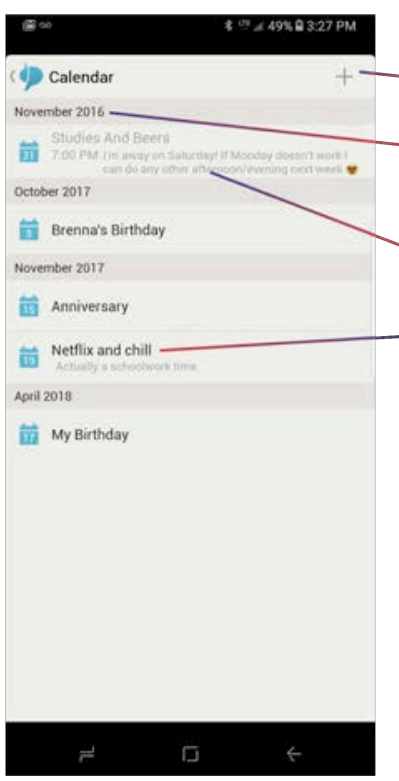

Separating months vertically runs the risk of having too much
dense type taking up all the space on the screen. There is no basis. Unsure as to why there is no option

weekly or monthly basis as well.

Aperil 20 


\section{SWOT Analysis (cont.)}

\section{Steady}

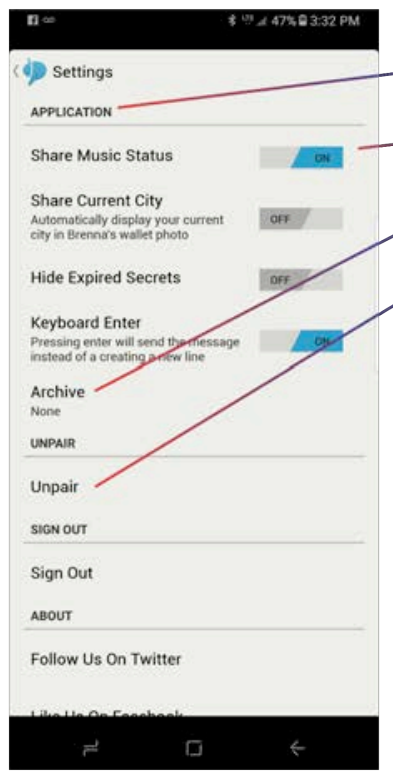

Application Settings

Separating different settings into groups with headers is a good looking for quicker.

Share music status is an interesting idea. Hypothetically, this would llow users to sync up apps ine Soundloud, Spotify or Google colld not figure out how to get this to work

Archive all content from the application. This would be extremely useful for merging all media and messages into one convenient .zip

Unpair in the event that a relationship does not work out, and a user
wishes to pair with another person without having to reinstall the wishes to pair with another person without having to reinstall the application.

Other settings not shown on this screen due to space include entering a securty passcode for opening the app, adjusting
notification settings and links to the app team's social media pages.

\section{Steady}

\section{Strengths}

- Upon tapping to expand, large area dedicated to partner's basic info and profile photo

- Decently organized UI, easy to comprehend so that users don't get stuck or run into dead end

made much easier

Insert

- Adopt FourSquare iconography to make businesses stand out in maps -

- Add dates and reminders that have options to repeat

- Seatings sreken downisto gize experience

- Adjust settings for your partner's info as well as your own

- Ability to unpair and repair the application with a different user

\section{Opportunities}

- Shared/synced media opens up a ton of opportunities for watching TV shows together while video chatting with an application like - Select your favorite photo of your partner to display as their profile photo - he or she will have the option to do the same according to his or her preferences

(liked shared Google Docs), such as journal entries and games el to an event or activity

- Insert a variety of media into conversation feed, making the screen pop with varied and interesting content
SWOT Analysis

Couple

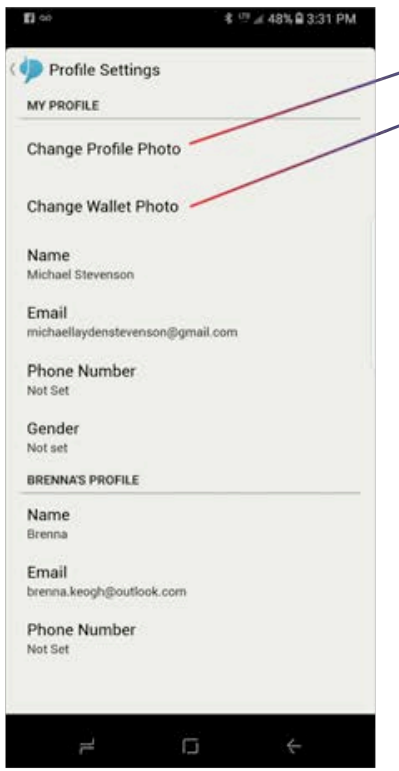

Profile Settings

Adjust profile settings such as contact information and profile

Partner's photo is described as "Wallet Photo", meaning that users can not only select a photo to represent their own accounts, but

\section{Weaknesses}

UI heavily borrowed from FourSquare feels uninspired, and has inconsistencies in different sections Redundancies with a few buttons and menus, - Not all insina lew spots, showing clipped text A few settings might be unnecesssary, such as selecting a gender - doesn't seem to have any particular use Some buttons are inconsistently designed

\section{Threats}

Trying to replicate all these features may be unnecessary, overcomplicating the app and bogging down Steady's appe 政 Segultings section could beco 


\title{
Site Map
}

\section{Steady}

\author{
Site Map/Content Inventory
}

HOME
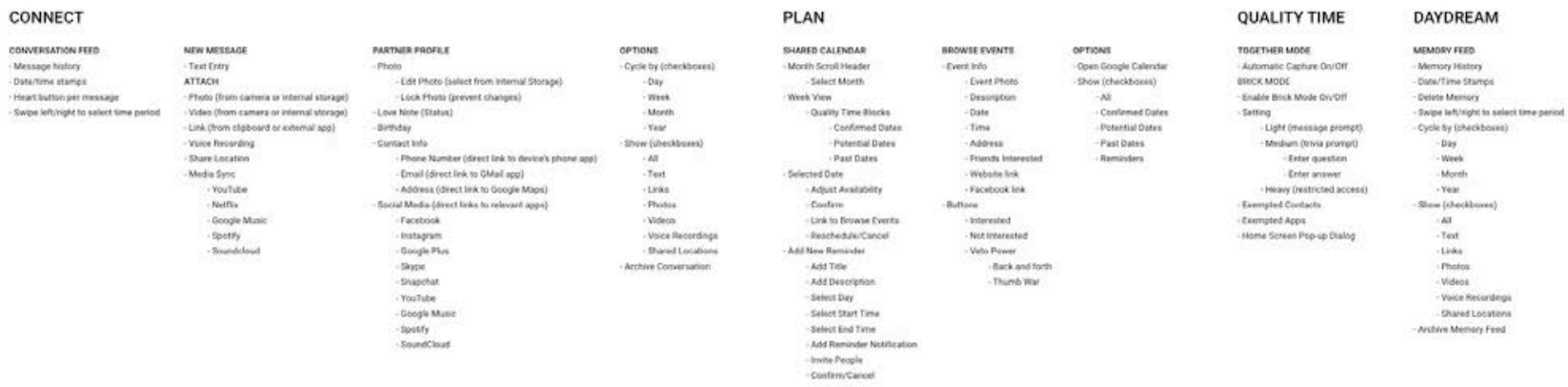

\section{Wireframes}

Steady

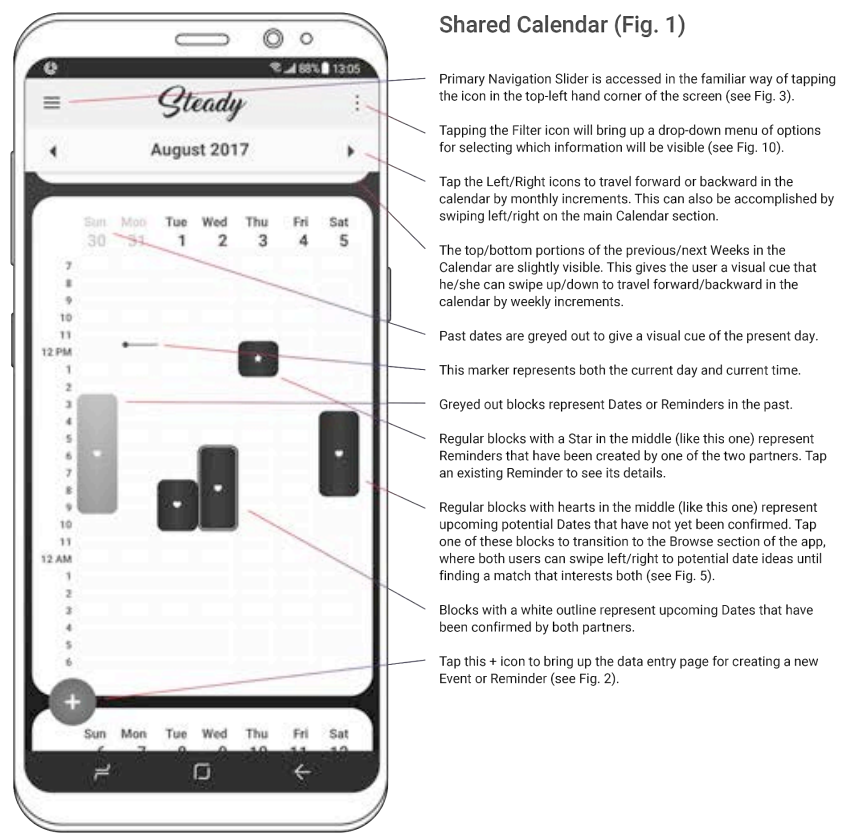

\section{Wireframes}

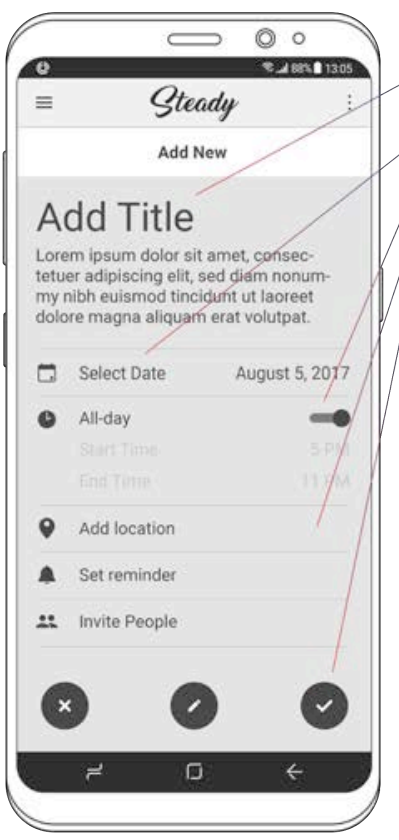

New/Edit Event (Fig. 2)

Tap to edit the title and description of the new reminder. Text field and keyboard will reveal from the bottom of the screen upon

Tapping on each setting will reveal a pop-up dialog box wit settings for adjusting values such as dates and times.

Tap this toggle switch to alternate between all-day activities, and th determined start and end times.

The link for adding a location will open a dialog box containing the Google Maps API for simple integration.

The icons at the bottom are for discarding or confirming changes as well as editing existing items. The Edit button in the middle would not be visible in this particular case, as this is a new Reminder. In the case of opening an existing Reminder for editing. 


\section{Wireframes (cont.)}

\section{Steady}

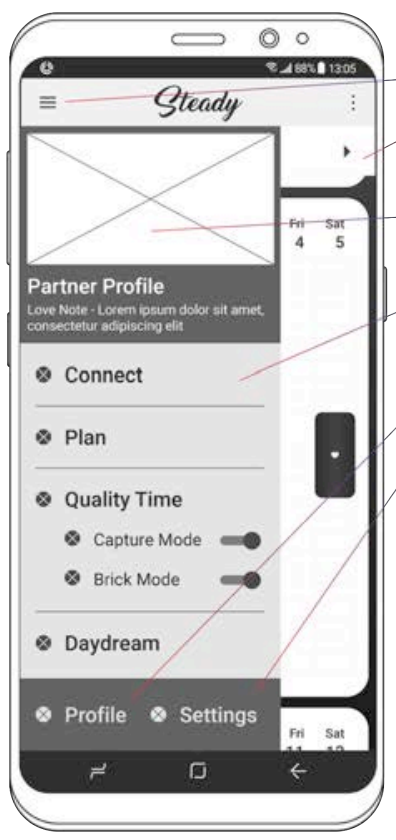

\section{Wireframes}

Navigation Slider (Fig. 3)

The Primary Navigation Slider appears from the left edge of the screen when the Nav icon (top-left) is tapped.

Whichever section of the app a user was viewing before openin (underneath the slider overlay). A user can exit the Nav and return

The first section of the Nav is Partner Profile, which simply contain a profile Image, Name and Love Note associated with a user's partner. Tapping anywhere inside this section wi
directly to his/her partner's Profile (see Fig. 4). Below this are links to the various other sections of the app.
Tapping on any one of these will take the user to that section. In the case of Capture Mode and Brick Mode, tapping the text will take the user to that section's settings,
turn this functionality on or off.

Takes the user to his/her own Profile settings, which make up wha s/her partner will see on their end.

Miscellaneous Settings.

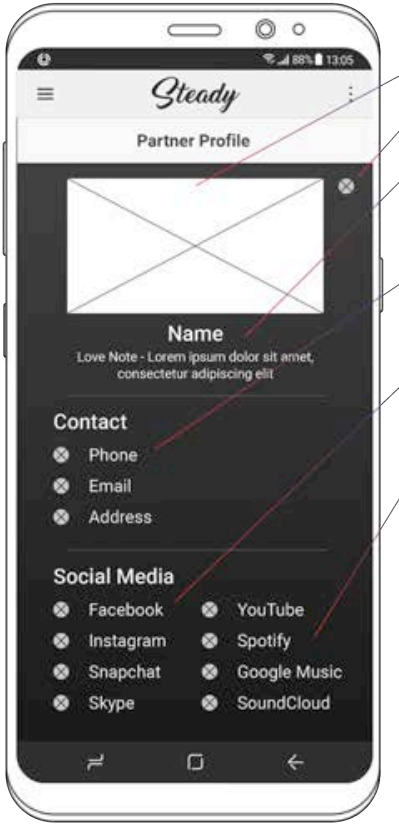

Partner Profile (Fig. 4)

The top section contains the same basic info that was shown as
part of the Primary Nav (see Fog. 3). part of the Primary Nav (see Fog.

Tapping the Edit icon allows a user to select a new photo of his/her partner to display.

This Love Note (tentative name) allows a user to leave a somewhat-hidden message for hish her partner, displayed here,
which can be changed at anytime what would be displayed here for example, would have been entered by this user's partner on the other end

Basic Contact info is displayed here. Tapping each will bring up the most relevant link. For example, tapping the Phone icon will prompt the Address icon will open up the destination in Google Maps, for quick access to directions of public transportation routes

Relevant Social Media links are displayed here, and tapping each
will take the user directly to his/her partner's profile on that Social Media site's server. This simfly keeps all the links together in one place, to a

For the media-consumption-related links (ex. YouTube), if a user's parther is cuntenly logged to tha plator (ex. Waching a video. the same timestamp in the same media. This allows partners to share viewing experiences across vast distances.

\section{Wireframes}

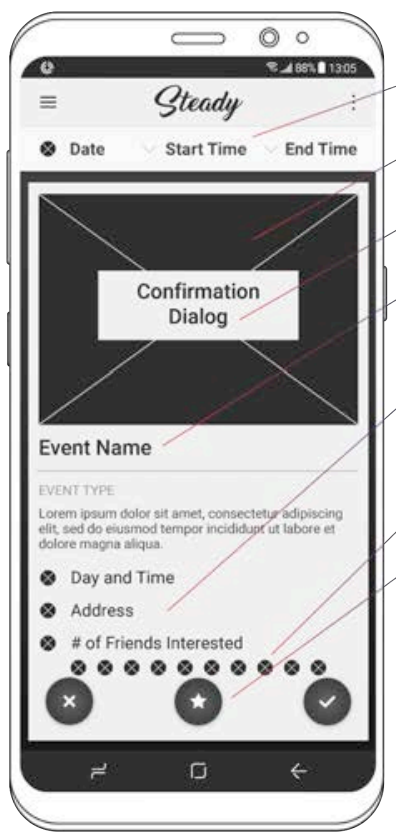

Swiping Potential Dates (Fig. 5)

Currently selected Date and Start/End Times are located at the top of the screen. Tap any
adjusting these values.

Event photo is displayed prominently. Swipe anywhere in the entire Event section to
selected event.

This Confirmation Dialog fades in as a user swipes left tor right on an Event, show

Event Name, Type (Nightlife, Sports, Art Exhibit, etc.) and a short description are provided here. Tapping on the Event Name will bring the Facebook Event page (opens the Facebook app externally and

Links for Day and Time, Address and Interested Friends provide additional useful information for determining whether an event is
feasible or desirable. Tapping these will either bring up a dialog box (such as displaying a list of a user's Interested Friends) or a promp asking the user if he/she would like to be directed to an external
application (such as opening the Address in Google Maps).

Thumbnail photos for Interested Friends pro
feedback without having to tap this section.

These icons have the same functions as swiping on the Event ection. The Star icon represents a user's Veto Power. Veto Power allows user to express great interest in an event, in the hopes that his/her partner will oblige. Once a user has activated Veto Power on


separate Event in the future. directs user to the appropriate link).

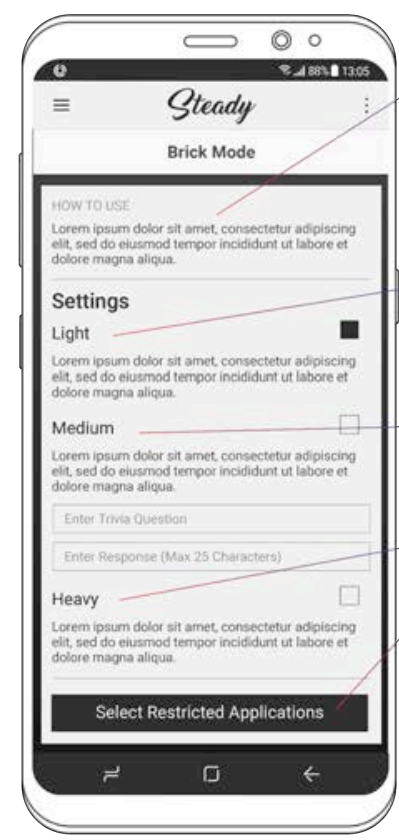

Brick Mode Options (Fig. 6)

Brick Mode helps users make a concerted effort to keep their mobile devices at bay while spending quality time together. Steady will implement NFC technology to determine when partners are
close proximity based on the locations of their smartphones, therefore there is never any need to activate Brick Mode manually at the beginning of a date. It can be turned off at any time from the
Primary Navigation Menu (see Fig. 3). Within the section itself are 3 settings users can choose from to determine which option (if any) works best for them. It is worth noting that whichever setting is ante setricted or tampered whin any way for

The Light setting does not pose any restriction on a user using his/her phone initially, but will simply take note of how often a use is checkn's (5 times in 1 hour for example). a coloffully worded

message will prompt the user. This message will inform the use of his/her excessive phone usage, along with a reminder of good date etiquette.

The Medium setting prevents the user from accessing his/he phone (or problematic applications) until he/she can answer a
simple trivia question that has been entered in advance by his/he partner. The goal with this option is to stimulate light banter whe

The Heavy setting prevents the user from accessing his/her phone simultaneously.

In future versions of the app, after sufficient data and feedback has been obtained, a user can this button to check boxes in a
drop-down list of installed apps. This allows a user to sin prevent his sher partner from as. preven as Snapchat, Instagram or a sports app) while spending
(such as quality time together 


\section{Wireframes (cont.)}

\section{Steady}

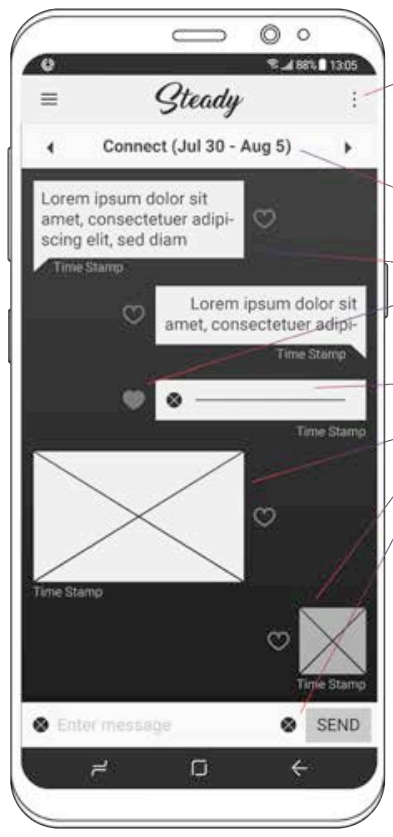

\section{Wireframes}

Connect (Fig. 7)

Tapping this icon reveals a drop-down list of options and filters,

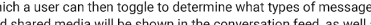
the desired duration of time from which to pull media. Depending on the types of media shown, the layout of the screen may change The current time frame is displayed in this banner, along with arrow
icons that inform the user he/she can swipe left or right to travel quickly through time periods.

A simple text message with a time stamp underneath.

This filled-in heart icon indicates a messge/post has been tapped and hiked by a user, and was added to the Daydream memory wall outlined hearts, which have not yet been liked in this example.

An attached voice recording with appropriate icon. A user can tap

he icon to begin playing the recording.

An attached photo.

An attached emoji.

In the text entry field, we see two icons - the left for attaching media ther for attaching emojis. Tapping to attach media pulls us naditional row of icons representing the various types of media hat can be attach

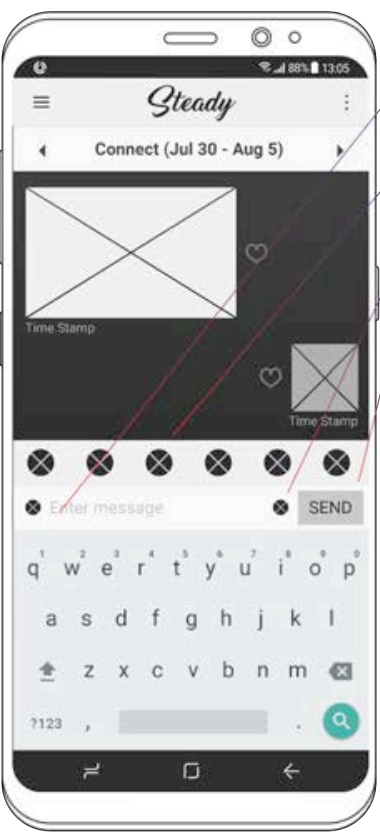

Connect With Keyboard (Fig. 8)

Upon tapping inside the text-entry field, the keyboard will scroll u the Connect conversation window up as well to compensate leaving the most recent messages/media visible on the screen.

Icons for the various types of media that can be attached to message. Tapping on one of these will open up a new dialog a Record button to begin recording a Voice Message.

Tapping the Insert Emoji icon will cause the keyboard to switch to the device's grid of available Emojis.

Tap Send to send a message.

\section{Steady}

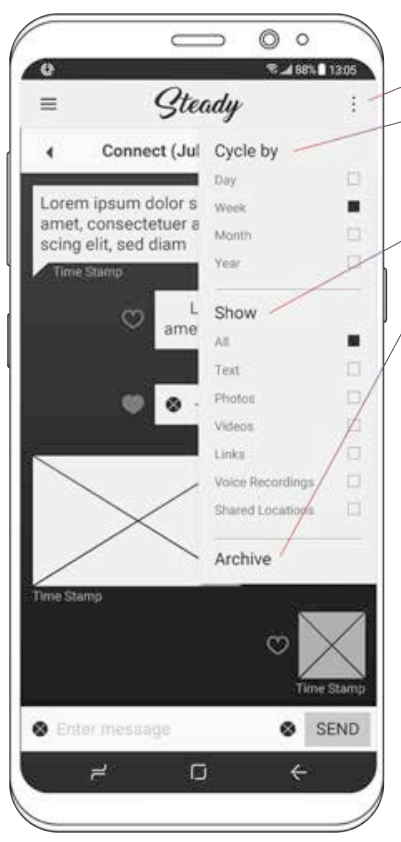

\section{Wireframes}

Connect With Filter (Fig. 9)

Tapping the Filter icon causes this drop-down menu to appear.

Cycle By allows the user to determine the unit of time he/she

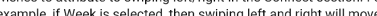
the conversation history back or forward one week in time - This llows the user to quickly scan through long periods of time to find a specific message, media or link

The Show filter list allows users to determine which types of messages and media they wish to

Tap the Archive button to bring up an additional dialog box, prompting the user whether to export an Archive (.zip file) of a

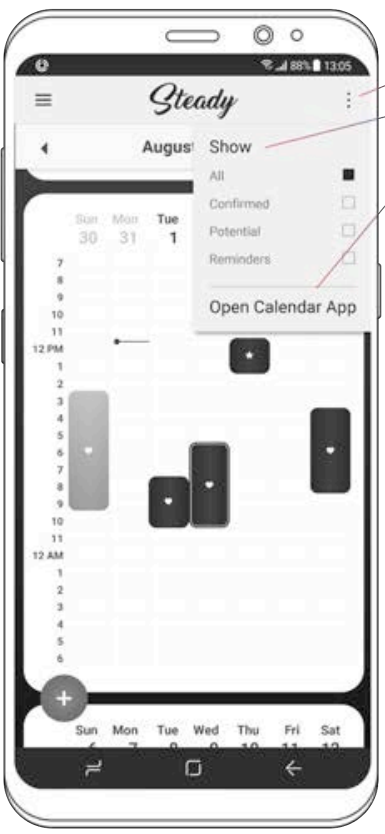

Calendar Options (Fig. 10)

Tapping the Filter icon causes this drop-down menu to appe The Show filter list allows users to determine which types of any unwanted clutter.

Tap the Open Calendar App button to bring up an additional dialoo box, prompting the user whether to open his/her external calend 


\section{Moodboards}

Tropical Sunset

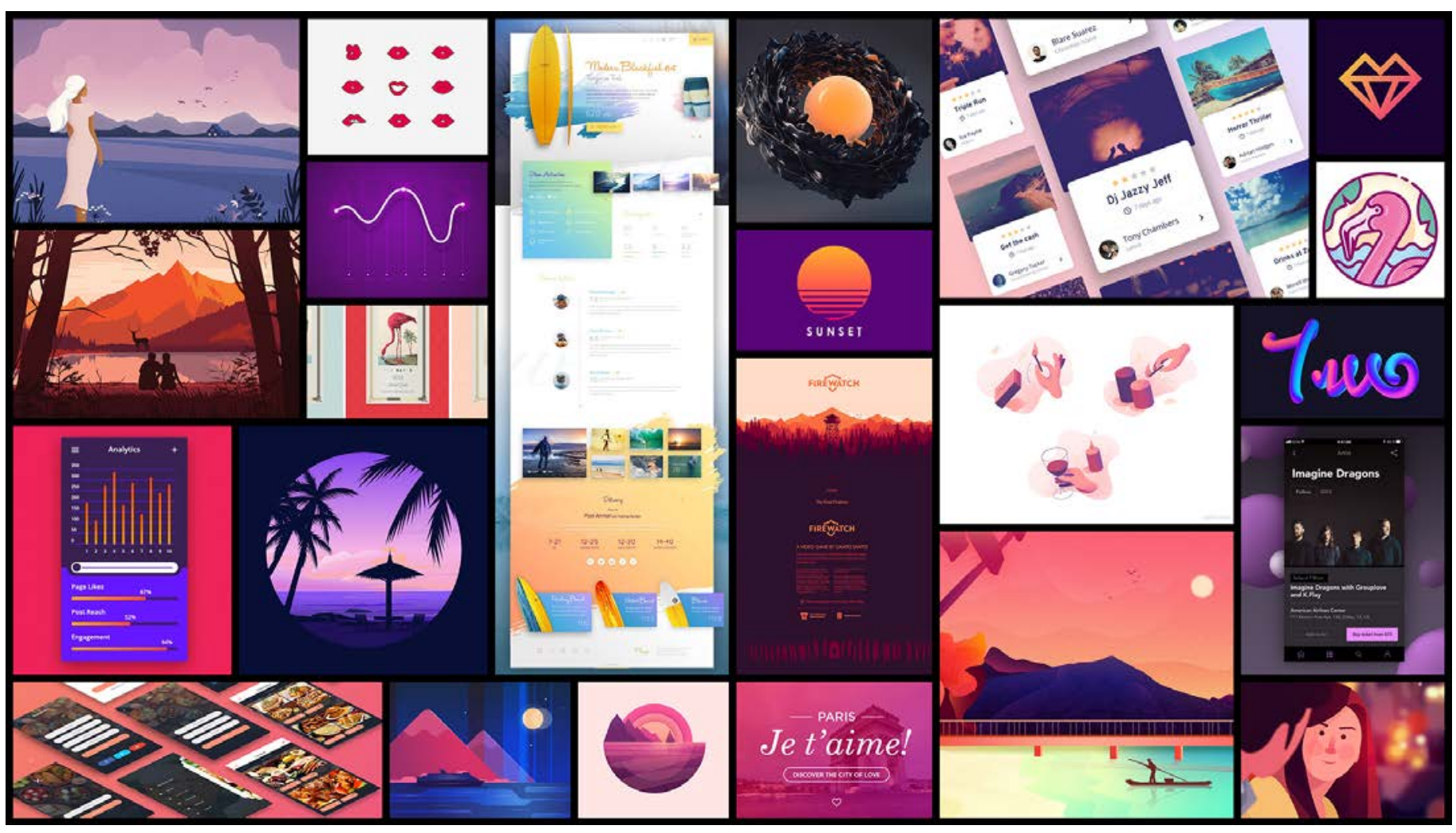




\section{Moodboards (cont.)}

Fragrance \& Lingerie

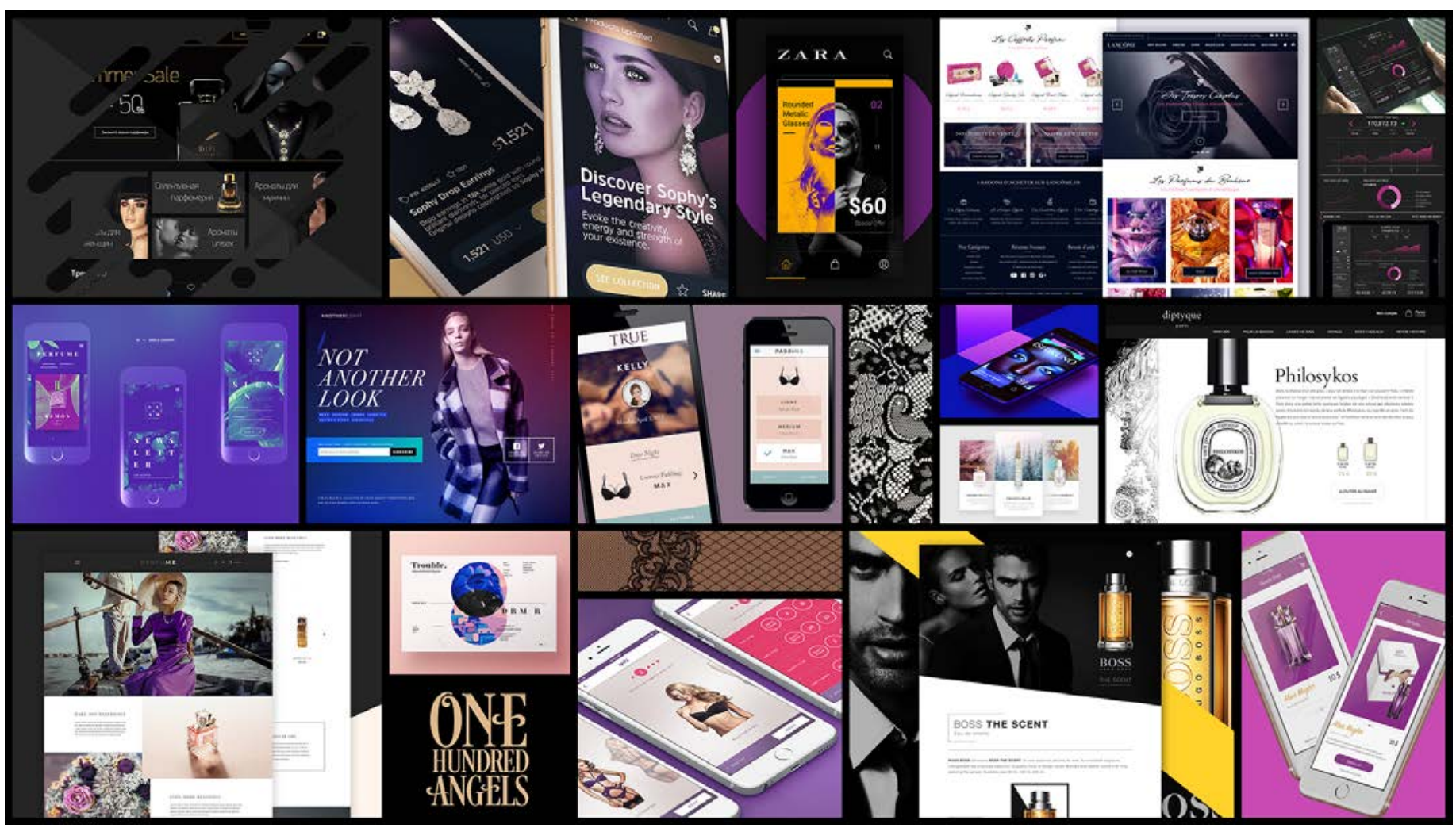




\section{Moodboards (cont.)}

Spring Wedding

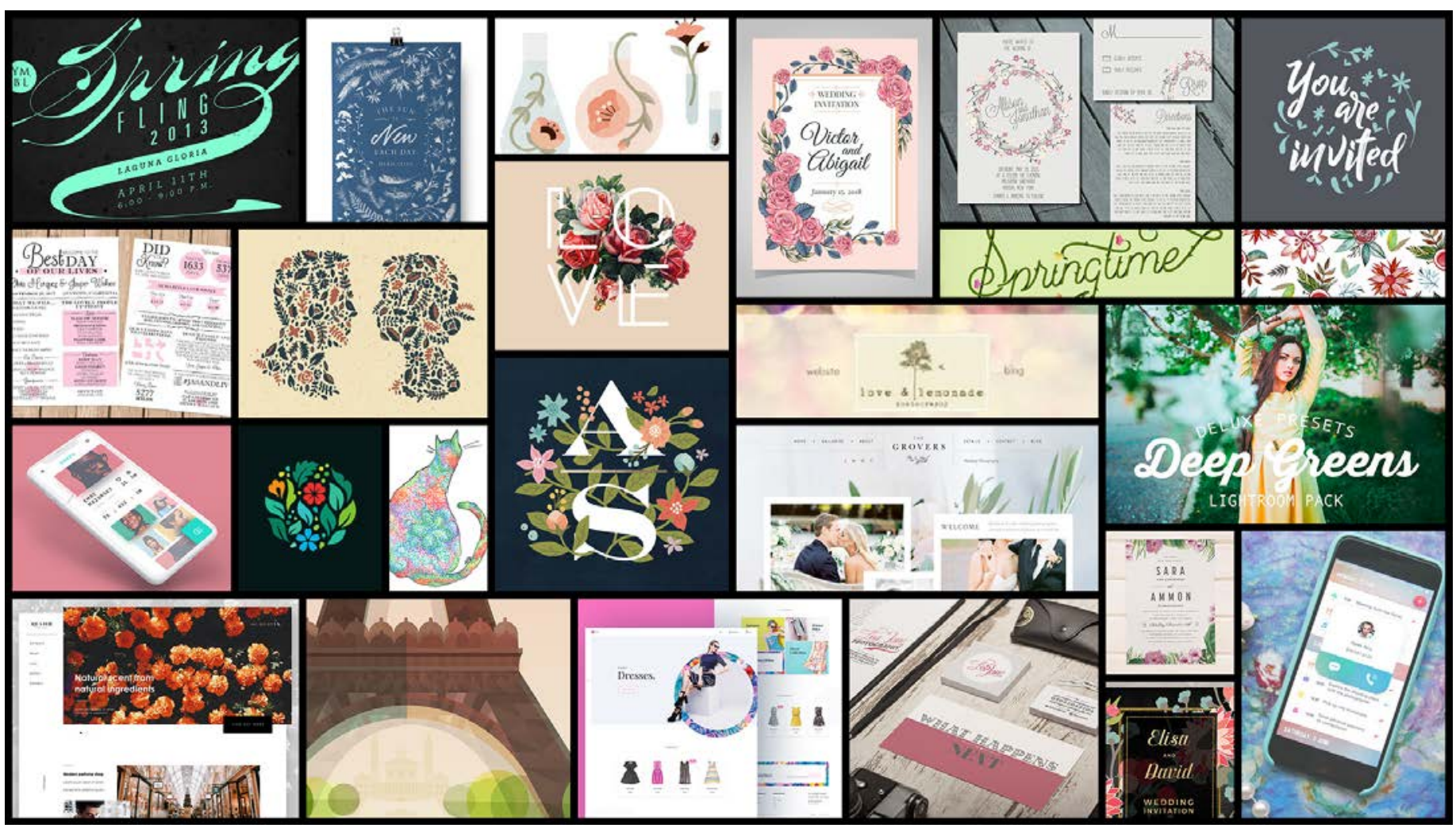




\section{Style Guide}

\section{Steady}

Brand Colours

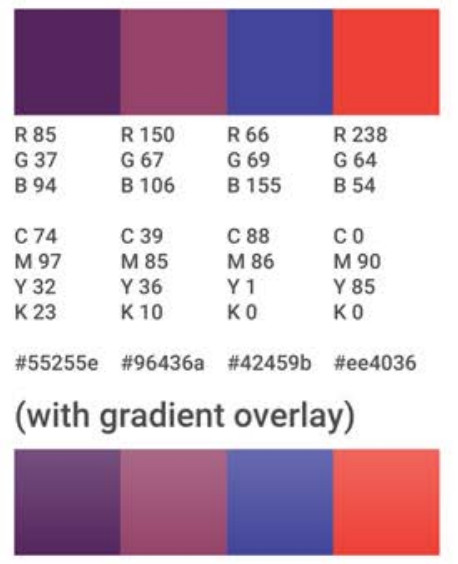

\section{Style Guide}

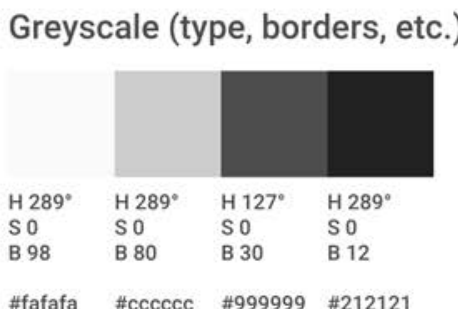

\#fafafa \#ccccc \#999999 \#212121

\section{Buttons/CTA's}

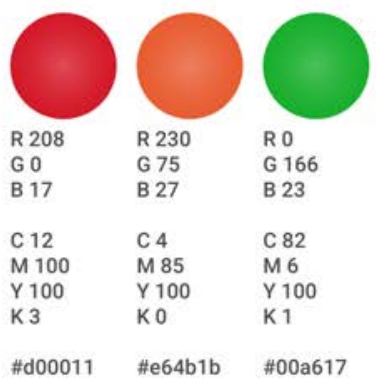

Thinking of Betty headline type

Roboto Medium Paragraph Type

\section{Logo \& Wordmark}
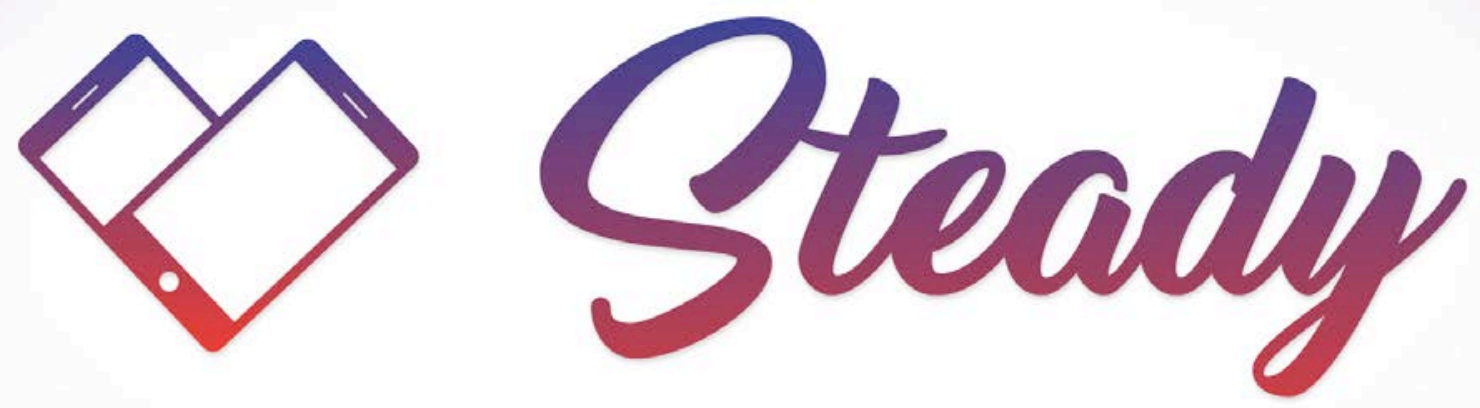


\section{Comps}

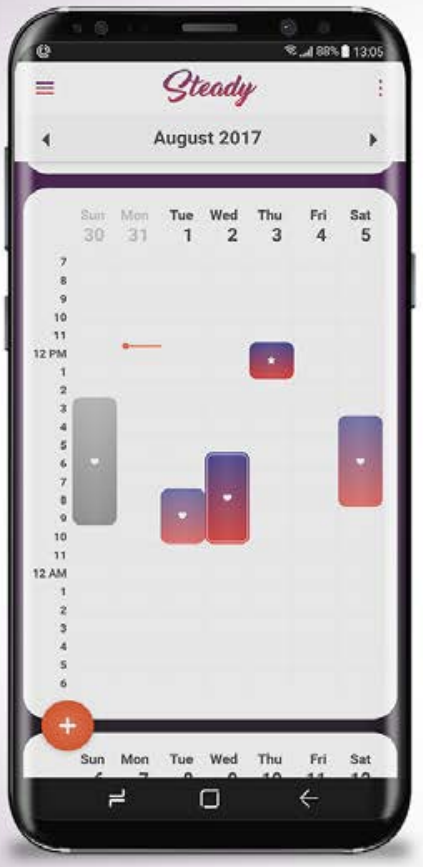

\section{Efficient Planning}

One of the primary obstacles faced by those in long-distance relationships is managing schedules and finding time to spend together. However, privacy is often a concern which keeps many couples from using a shared calendar application.

Steady circumvents this issue by only displaying the optimal times for both partners to get together, based on an algorithm that takes both schedules into account and encourages compromise.

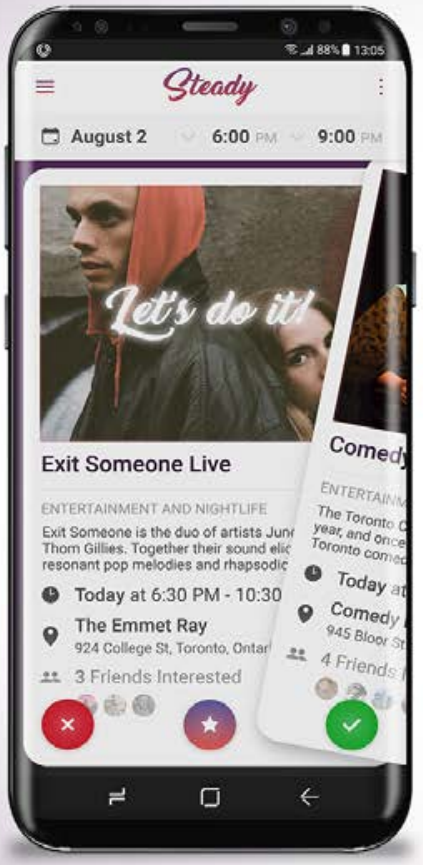

\section{Keep Things Exciting}

Once a day and time has been agreed upon, users can swipe left or right in response to potential date ideas, which are populated by aggregating partners' Facebook Likes. If both partners swipe right to the same date idea, a match occurs much like in popular dating apps, and links to purchase tickets are made instantly available.

This portion of the application was designed as a response to the growing popularity of casual dating apps, giving couples a more constructive way to swipe. 


\section{Comps (cont.)}
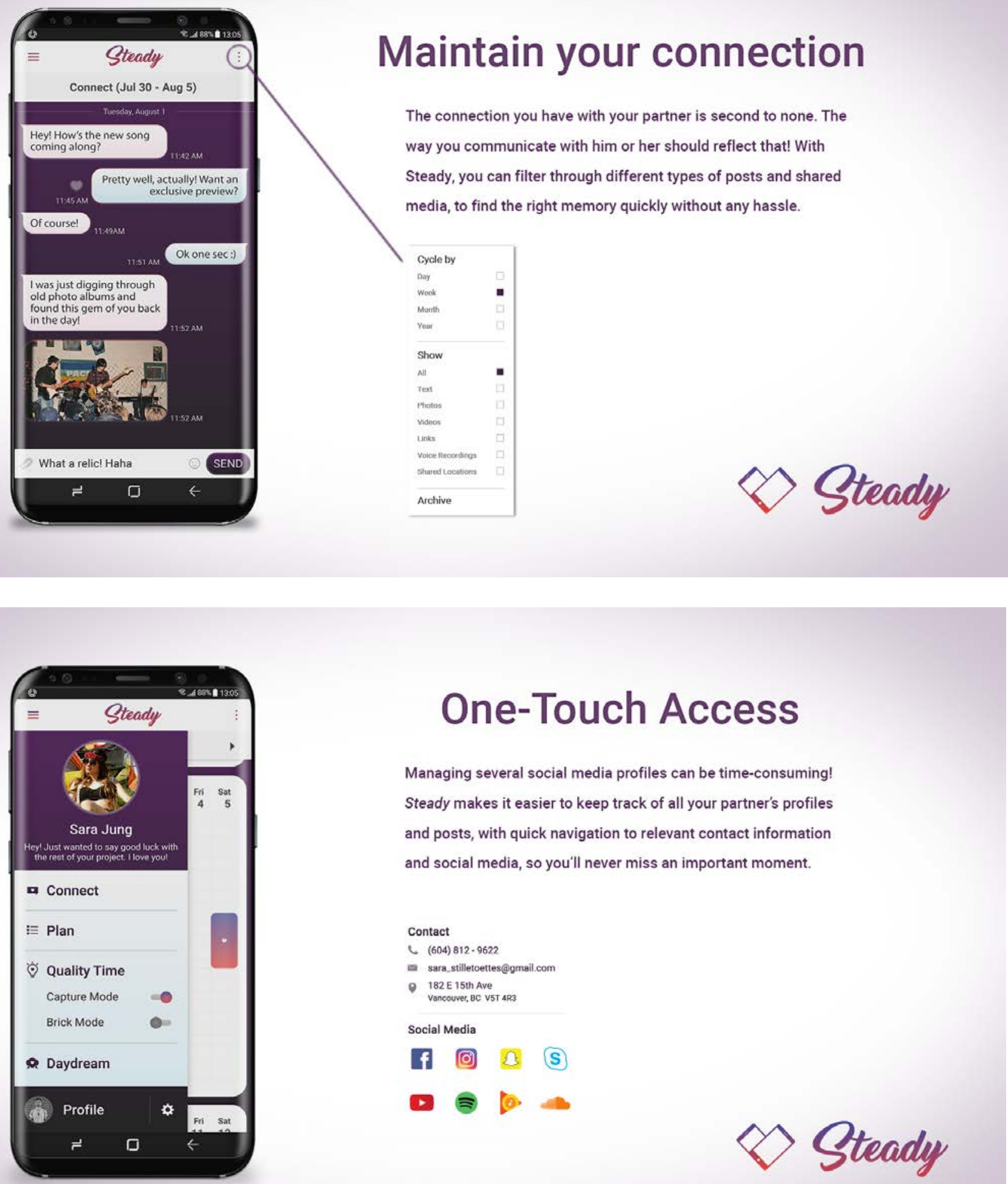

\section{One-Touch Access}

Managing several social media profiles can be time-consuming!

Steady makes it easier to keep track of all your partner's profiles and posts, with quick navigation to relevant contact information and social media, so you'll never miss an important moment.

Contact

C. (604) 812.9622

a sara_stilletoettes@gmail.com

- $182 \mathrm{E} 15$ th Ave

Social Media

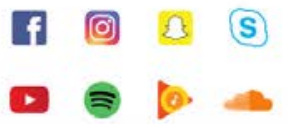


Comps (cont.)

(). ร..nl $88 \% \square 13: 05$

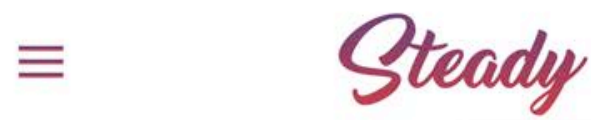

Sara Jung

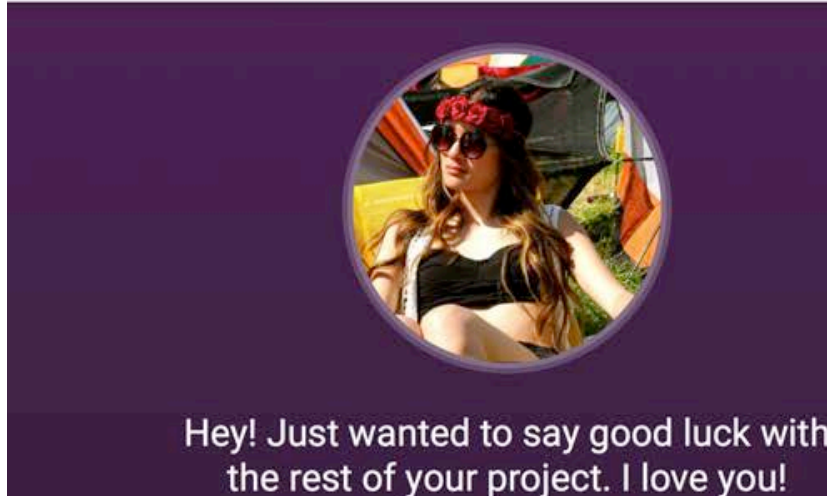

Contact

C (604) 812 - 9622

\. sara_stilletoettes@gmail.com

- 182 E 15th Ave Vancouver, BC V5T 4R3

\section{Social Media}
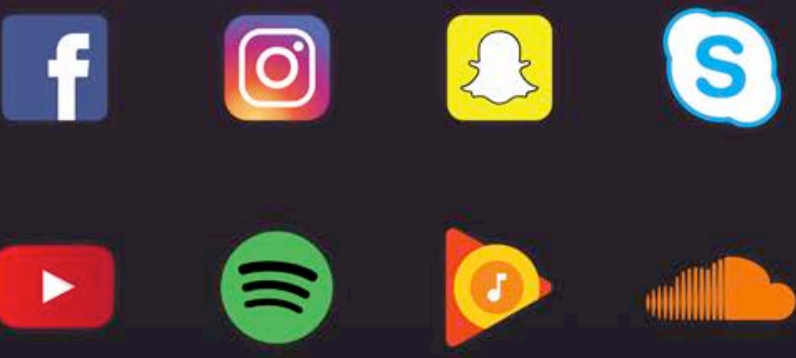

$\rightleftharpoons$

Ð

$\leqslant$ 


\section{References}

Appel, H., Crusius, J., \& Gerlach, A. (2015). Social comparison, envy, and depression on Facebook: A study looking at the effects of high comparison standards on depressed individuals. Journal of Social and Clinical Psychology, 34(4), 277-289, Retrieved:

https://www.researchgate.net/publication/274067560_Social_Comparison_Envy_and_Depression_on_Facebook_A_Study_L ooking_at_the_Effects_of_High_Comparison_Standards_on_Depressed_Individuals

Blue Bite (2017, June 22). The promising future of NFC. Medium, Retrieved: https://medium.com/@BlueBite/thepromising-future-of-nfc-d172f6905b7b

Boyd, M. (2013, December 13). API Aggregation: Why it matters and eight different models. Programmable Web, Retrieved: https://www.programmableweb.com/news/api-aggregation-why-it-matters-and-eight-different-models/2013/12/13

Coyne, S. M., Stockdale, L., Busby, D., Iverson, B., \& Grant, D. M. (2011). "I luv u:)!”: A descriptive study of the media use of individuals in romantic relationships. Family Relations, 60(2), 150-162, Retrieved:

http://www.jstor.org.ezproxy.lib.ryerson.ca/stable/41236755?pq-origsite=summon\&seq=1\#page_scan_tab_contents

Dawley, S. (2016, October 24). A long list of Facebook statistics - and what they mean for your business. Hootsuite, Retrieved: https://blog.hootsuite.com/facebook-statistics/

Finkel, E., Eastwick, P., Karney, B., Reis, H., \& Sprecher, S. (2012). Online dating: A critical analysis from the perspective of psychological science. Psychological Science in the Public Interest, 13(1), 3-66, Retrieved:

http://journals.sagepub.com.ezproxy.lib.ryerson.ca/doi/full/10.1177/1529100612436522

Grinols, A. B. \& Rajesh, R. (2014) Multitasking with smartphones in the college classroom. Business and Professional Communication Quarterly, 77(1), 89-95, Retrieved:

http://journals.sagepub.com.ezproxy.lib.ryerson.ca/doi/full/10.1177/2329490613515300\#articleCitationDownloadConta $\underline{\text { iner }}$

Henline, B. H. (2006). Technology use and intimacy development in committed relationships: Exploring the influence of differentiation of self. Unpublished doctoral dissertation, Texas Tech University, Retrieved:

https://www.researchgate.net/publication/265081113_TECHNOLOGY_USE_AND_INTIMACY_DEVELOPMENT_IN_COMMIT TED_RELATIONSHIPS_EXPLORING_THE_INFLUENCE_OF_DIFFERENTIATION_OF_SELF

Hertlein, K. M. \& Piercy, F. P. (2008). Therapists' assessment and treatment of Internet infidelity cases. Journal of Marital and Family Therapy, 34(4), 481-497, Retrieved: http://onlinelibrary.wiley.com.ezproxy.lib.ryerson.ca/doi/10.1111/j.17520606.2008.00090.x/abstract

Hilton-Dunne, Abii (2016, March 3). How to think about dating when you have Border Personality Disorder. Elite Daily, Retrieved: https://www.elitedaily.com/dating/borderline-personality-disorder-dating/1376748

Lyadova, K. \& Hughes, M. (2017). Dating Vandalized. Toronto, Ontario. Oddbee, Inc.

McCormack, M. (2015). 'The role of smartphones and technology in sexual and romantic lives.' Project Report, Durham University, Retrieved:

https://www.researchgate.net/publication/283071203_The_Role_of_Smartphones_and_Technology_in_Sexual_and_Roman $\underline{\text { tic_Lives }}$

Matyszczyk, C. (2015, May 7). Nearly Half Of Tinder Users Are Already With Someone, Says Study. CNet, Retrieved: https://www.cnet.com/news/nearly-half-of-tinder-users-are-already-with-someone-says-study/

Nahai, N. (2017, February 12). There's a dark side to dating apps - they won't help you find love for Valentine's Day. Independent, Retrieved: http://www.independent.co.uk/voices/dating-apps-valentines-day-tinder-bumble-grindralgorithms-dopamine-a7575996.html 
Notable Life (2015, November 26). 6 ways to date smart as a busy young professional. Notable Life, Retrieved: https://notablelife.com/6-ways-to-date-smart-as-a-busy-young-professional/

Pettigrew, J. (2009). Mobile text messaging and connectedness within close interpersonal relationships. Marriage \& Family Review, 45(7), 697-716, Retrieved:

https://www.researchgate.net/publication/233096873_Text_Messaging_and_Connectedness_Within_Close_Interpersonal_ Relationships

Prizant-Passal, S., Shechner, T., \& Aderka, I. M. (2016). Social anxiety and internet use - A meta-analysis: What do we know? What are we missing? Computers in Human Behavior, 62, 221-229, Retrieved: http://www.sciencedirect.com.ezproxy.lib.ryerson.ca/science/article/pii/S0747563216302680

Przybylski, A. \& Weinstein, N. (2012). Can you connect with me now? How the presence of mobile communication technology influences face-to-face conversation quality. Journal of Social and Personal Relationships, 30(3), 237-246, Retrieved: http://spr.sagepub.com/content/early/2012/07/17/0265407512453827.full.pdf+html

Rendón, F. (2015, July 25). 12 tips on how to maintain a healthy relationship while traveling for work. The Good Men Report, Retrieved: https://goodmenproject.com/featured-content/12-tips-on-how-to-maintain-a-healthy-relationship-whiletraveling-for-work-wcz/

Roberts, J. \& David, M. (2017). Partner phubbing: How cell phones impact romantic partnerships. Keller Center Research Report, 10(1), Retrieved: https://www.baylor.edu/business/kellercenter/doc.php/277897.pdf

Sam Chan, L. (2017). Who uses dating apps? Exploring the relationships among trust, sensation-seeking, smartphone use, and the intent to use dating apps based on the integrative model. Computers in Human Behavior, 72, 246-258, Retrieved: https://journals-scholarsportal-info.ezproxy.lib.ryerson.ca/details/07475632/v72icomplete/246_wudaetabotim.xml

Schade, L.C., Sandberg, J., Bean, R., Busby, D., \& Coyne, S. (2013). Using technology to connect in romantic relationships: Effects on attachment, relationship satisfaction, and stability in emerging adults. Journal of Couple \& Relationship Therapy, 12(4), 314-338, Retrieved: https://journals-scholarsportal-

info.ezproxy.lib.ryerson.ca/details/15332691/v12i0004/314_uttcirsasiea.xml

Smith, C. (2018, January 3). 45 Impressive Tinder Statistics. Expanded Ramblings, Retrieved: http://expandedramblings.com/index.php/tinder-statistics/

Strapagiel, L. (2016, February 12). This Toronto woman hired someone to manage her online dating profiles and it's kinda working. BuzzFeed, Retrieved: https://www.buzzfeed.com/laurenstrapagiel/why-arent-we-all-doingthis?utm_term=.isq710D1M\#.foWn3p531

Tackie-Ofosu, V. (2015). Parents' experiences with digital lifestyles of young adults: A Ghanaian perspective. Journal of Family and Consumer Sciences, 107(2), 32-40, Retrieved:

http://search.proquest.com.ezproxy.lib.ryerson.ca/docview/1708016629?pq-origsite=summon

Thomsen, D. (2012). Why human-centered design matters. Wired, Retrieved.

https://www.wired.com/insights/2013/12/human-centered-design-matters/

Turkle, S. (2011). Alone Together: Why We Expect More from Technology and Less from Each Other. New York, NY: Basic Books, Retrieved: https://apps.carleton.edu/curricular/aiseminars/assets/Sherry_Turkle_book.pdf

Vendeville, G. (2016, January 28). Woman, 30, hires dating profile manager from Craigslist to help her find romance. The Star, Retrieved: https://www.thestar.com/business/2016/01/28/woman-30-hires-dating-profile-manager-fromcraigslist-to-help-her-find-romance.html

Weiss, S. (2016, May 10). Why we swipe right and then ignore our Tinder matches. Bustle, Retrieved: https://www.bustle.com/articles/157940-why-we-swipe-right-and-then-ignore-our-tinder-matches

Wells, J. (2014, February 14). 7 reasons why I use InVision for rapid prototyping. Medium, Retrieved: https://medium.com/@mrjeremywells/7-reasons-why-i-use-invision-for-rapid-prototyping-ed1c33d5b86 
Wong, B. (2017, November 1). This trend is ruining relationships (and you're probably guilty of it). Huffington Post, Retrieved: http://www.huffingtonpost.ca/entry/phubbing-is-probably-ruining-your-

relationship_us_59f9fc07e4b046017fb07e32

Wong, B. (2016, December 14). There's a new trend in one-night stands (and you're probably doing it). Huffington Post, Retrieved: http://www.huffingtonpost.ca/entry/the-new-trend-in-one-night-stands-youve-probably-

experienced_us_584b15a4e4b04c8e2bafe2f2 